\title{
Accessibilité aux soins des femmes et des enfants, cas de trois quartiers squattés de la ville d'El-Mina (Liban)
}

\section{Elias Alissar}

\section{Q OpenEdition}

1 Journals

Édition électronique

URL : http://journals.openedition.org/rfst/679

DOI : $10.4000 /$ rfst.679

ISSN : 2492-3672

Éditeur

Espaces et SOciétés (UMR 6590)

Référence électronique

Elias Alissar, «Accessibilité aux soins des femmes et des enfants, cas de trois quartiers squattés de la ville d'El-Mina (Liban) », Revue francophone sur la santé et les territoires [En ligne], Miscellanées, mis en ligne le 21 juillet 2016, consulté le 06 avril 2021. URL : http://journals.openedition.org/rfst/679 ; DOI : https://doi.org/10.4000/rfst.679

Ce document a été généré automatiquement le 6 avril 2021.

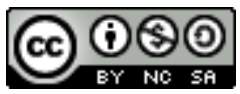

La Revue francophone sur la santé et les territoires est mise à disposition selon les termes de la Licence Creative Commons Attribution - Pas d'Utilisation Commerciale - Partage dans les Mêmes Conditions 4.0 International. 


\title{
Accessibilité aux soins des femmes et des enfants, cas de trois quartiers squattés de la ville d'El-Mina (Liban)
}

\author{
Elias Alissar
}

\section{Introduction}

1 La ville d'El-Mina fait partie de l'Union des municipalités d'Al-Fayhaa ${ }^{1}$ dont la municipalité principale est Tripoli, capitale du Liban Nord. Elle s'étale sur $13 \%$ de la surface de l'Union et regroupe 53,201 (Baghdadi S., 2005) habitants avec une densité humaine équivalente à 142 personnes / ha. Cette ville dont le tissu urbain a fini par rejoindre les limites de la ville de Tripoli est caractérisée par sa situation géographique côtière et par sa forme de péninsule entourée des trois bords par la mer Méditerranée. La ville d'El-Mina est distante de $3 \mathrm{~km}$ à l'ouest de Tripoli ; les deux villes sont reliées par trois routes principales : El-Mitein, Azmi, et Mina.

2 Loin des restaurants et sites touristiques de cette ville côtière, se trouvent des quartiers défavorisés souvent inconnus par une grande majorité des habitants des autres secteurs de la ville. L'expansion aléatoire du tissu urbain a abouti à l'émergence de quartiers squattés suite à l'occupation illégale des terrains et des bâtiments vacants. Squattériser (Larousse p. 977) ou squatter est le fait d'« occuper un logement vide». Mais dans la littérature anglo-saxonne le squatteur (squatter) est « une personne qui s'installe sur un terrain sans titre juridique ». Le terme de "squatter settlement» ou quartier squatté a été développé dans les années 1960 par C. Abrams et réutilisé dans les années 1970 lors de la première Conférence internationale sur l'Habitat tenue à Montréal. "A squatter settlement therefore, can be defined as a residential area which has developed without legal claims to the land and/or permission from the concerned authorities to build; as a result of their illegal or semi-legal status, infrastructure and services are usually inadequate". 
3 Dans les pays en développement, on applique le terme pour l'occupation illégale d'un terrain pour y construire un logement en général sommaire (Merlin P., Choay F.; 1996). Dans la ville d'El-Mina le squat associe terrains et immeubles illégalement occupés, quelquefois dans un même espace.

4 Ces territoires, Hay El-Tanak, Haouch et El Masaken El-Chaabiyah abritent des populations dont la grande majorité appartient à des statuts socio-économiques précaires.

5 Les territoires informels de la ville d'El-Mina sont apparus vers la fin du $19^{\text {ème }}$ siècle. Il s'agit de quartiers de taille moyenne qui sont répartis notamment sur la rive ouest de la ville, à l'exception de Khan El-Tamathili, localisé sur la côte est. Les habitants de ces espaces sont menacés par l'évacuation forcée suite aux réclamations des propriétaires réels qui demandent une récupération de leurs terrains illégalement occupés.

6 Les observations initiales ont révélé que les quartiers squattés mentionnés n'ont pas des niveaux de morbidité plus élevés que les autres quartiers de la ville. Cette situation se traduit par une absence des problèmes de santé qui suscitent des interventions urgentes.

7 Cependant, les quartiers squattés présentent de multiples vulnérabilités qui menacent la santé et le bien-être des populations qui y résident. En effet, " le chômage, la pollution, la médiocrité du logement, les inégalités, la pauvreté, les milieux malsains, les difficultés d'accès au travail, aux biens et aux services, le stress et le manque de cohésion sociale: tout cela affecte la santé et le bien-être des personnes "(OMS, 2000). En particulier, plusieurs risques liés à l'habitat précaire et insalubre provoquent des pathologies diverses, souvent graves, chez les habitants permanents dans ces territoires.

8 Nous avons remarqué que les populations occupant les territoires squattés ciblés de la ville d'El-Mina ont pu surmonter les faiblesses du milieu. Ainsi, ils arrivent à survivre avec un moindre impact sur leur bien-être sanitaire. Ceci nous a poussés à nous intéresser à cet espace peu étudié particulièrement à propos de la santé des femmes au cours de la phase périnatale et la santé des enfants au cours de leurs 5 premières années de vie. Ces deux groupes étant les plus fragiles parmi les habitants.

9 Dans son rapport, publié en 2009, sur la santé des femmes dans le monde, l'OMS affirmait que " la santé revêt une importance centrale pour les femmes mais aussi pour leurs enfants qu'elles seront amenées à porter. Ainsi, il importe de souligner que se préoccuper de la santé des filles et des femmes aujourd'hui représente un investissement pour le présent mais également pour les générations futures (OMS, 2009, p.3) »Pour évaluer la santé de ces dernières plusieurs indicateurs sont à étudier en commençant par le suivi au cours de la grossesse.

10 Au Liban, des études traitant les indicateurs de santé périnatale et la santé des enfants de moins de 5 ans sont souvent des études descriptives effectuées sur la totalité du pays qui ne prennent pas en considération les déterminants urbains et socio-économiques spécifiques de chaque territoire. Parmi ces études le projet PAPFAM (Pan Arab Project for Family Health) mené en 1996 qui a ciblé la totalité du territoire Libanais. Ces mêmes indicateurs, en plus de plusieurs autres, ont été repris dans une étude similaire en 2004. En l'an 2000, le conseil des ministres en collaboration avec le fond des Nations unies pour les enfants réalisèrent une étude portant sur la mesure des indicateurs de santé des enfants au Liban. Les résultats de la première phase du projet intitulé "MICS 2 " (Multiple Indicators Cluster Survey) ont été publiés en 2001 alors que la seconde phase 
« MICS 3 » a été diffusée en 2009 sur le site de l'administration centrale des statistiques. Dans celles-ci l'aspect géographique a été introduit dans la répartition des problèmes de santé selon les caza (district) sans établir les caractéristiques typologiques des quartiers. D'où l'intérêt de cet article dans lequel nous aborderons la problématique de la santé à l'échelle locale du quartier en prenant en considération les caractéristiques géographiques, économiques et sociales de chaque territoire.

11 Afin d'explorer l'état de santé des femmes et des enfants habitant les quartiers squattés de la ville d'El-Mina, une enquête a été menée auprès des ménages occupant ces territoires entre 2009 et 2010. L'enquête a permis la construction d'une base de données qui servira à l'explication de cet état de santé suite à l'évaluation de l'accessibilité aux soins nécessaires. Ainsi nous avons procédé au calcul de l'indice d'accessibilité se basant sur la matrice des écarts de l'indice d'accessibilité de Shimbel (Pumain D., Saint-Julien T., 1997 - voir plus loin ${ }^{\circ}$ )». Cette méthode a permis d'estimer en théorie quel pourrait être le centre de soin le plus accessible. Les résultats obtenus ont été ensuite comparés aux destinations réelles déclarées par les personnes enquêtées. En revanche, pour mieux comprendre les choix de centres de soins adoptés par la population étudiée, il aura fallu explorer comment fonctionne le système de soin dans la ville. D'où l'intérêt d'identifier les acteurs urbains de santé y existant. Cependant, quelle est l'importance accordée à la proximité géographique lors du recours aux soins? De plus, on peut aussi interroger le rôle que jouent les acteurs urbains identifiés dans l'offre de soins assurée aux populations les plus démunies. Ceuxci ont il créé un réseau de solidarité dans la ville d'El-Mina pour aider les habitants des quartiers squattés dans l'accessibilité aux soins nécessaires?

\section{Méthodologie}

\section{Enquête et échantillonnage}

12 Une enquête de terrain a été menée entre Novembre 2009 et Juin 2010 auprès de 139 femmes de 15 à 49 ans. Elles ont répondu au questionnaire révélant des informations sur leur santé et celles de leurs familles, en particulier la santé de leurs enfants de moins de cinq ans.

13 Dans un premier lieu, nous avons effectué un dénombrement des ménages de Hay ElTanak d'abord, puis de Haouch et d' El Masaken El-Chaabiyah . Le nombre de ménages et de logements dans ces quartiers varie constamment. Un logement est susceptible d'être divisé en deux unités pour abriter un enfant qui se marie ou un nouveau ménage qui vient habiter dans le quartier. Nous avons pris comme cible d'enquête les ménages représentés par les mères dont la grande majorité est en âge de procréation. Par conséquent, nous avons pu dénombrer $185^{2}$ ménages à Hay El-Tanak, 94 ménages à Haouch et 386 ménages dans le quartier d' El Masaken El-Chaabiyah (Tableau 1).

14 L'étude menée dans le cadre de cette recherche est transversale descriptive et l'échantillon étudié a été aléatoirement choisi. Ce dernier est équivalent à $21 \%$ de la totalité des ménages habitant ces espaces. Ainsi, 47 femmes de Hay El-Tanak , 23 femmes résidant à Haouch et 69 femmes habitant aux Masaken (Tableau 1) ont été simultanément interrogées, révélant des données sur l'ensemble du ménage dont elles représentent. 
Tableau 1 - Dénombrement des ménages habitant les quartiers squattés ciblés

\begin{tabular}{|l|l|l|l|}
\hline Nom du Quartier & $\begin{array}{l}\text { Nombre total de } \\
\text { ménages }\end{array}$ & $\begin{array}{l}\text { Nombre des ménages } \\
\text { enquêtés }\end{array}$ & $\begin{array}{l}\text { des ménages } \\
\text { enquêtés }\end{array}$ \\
\hline Hay El-Tanak & 185 & 47 & 25.4 \\
\hline Haouch & 94 & 23 & 24.5 \\
\hline $\begin{array}{l}\text { El Masaken El- } \\
\text { Chaabiyah }\end{array}$ & 386 & 69 & 17.9 \\
\hline Total & $\mathbf{6 6 5}$ & $\mathbf{1 3 9}$ & $\mathbf{2 0 . 9}$ \\
\hline
\end{tabular}

Source : Enquête Elias A. 2009 - 2010

En somme, la population totale enquêtée a porté sur 139 femmes en âge de procréation avec 111 enfants âgés de 5 ans et moins comme le montre le tableau ci-dessus.

Pour évaluer la santé périnatale les indicateurs étudiés sont: le suivi de la dernière grossesse et le lieu de suivi (figure 1), les naissances prématurées, les accouchements compliqués, les mortalités néonatales précoces et les décès après la première semaine de vie (les mortalités rapportées dans les trois ans précédant l'enquête).

Pour étudier la santé des enfants âgés moins de 5 ans, les indicateurs étudiés sont les symptômes de diarrhée, de fièvre et de toux au cours des trois mois précédant l'enquête. La santé des enfants est aussi évaluée par le respect accordé de la part des parents au calendrier des vaccins par rapport à l'âge de l'enfant. Sachant que les vaccins augmentent l'immunité contre des maladies pouvant être fatales. Cette partie du questionnaire comporte aussi des interrogations sur les comportements des mères suite à la survenue des infections de fièvre, de diarrhée et de toux ainsi que leurs préférences de lieu de recours aux soins pour leurs enfants.

18 L'échantillon au sein de chaque quartier a été choisi aléatoirement en commençant par la première unité résidentielle et continuant avec la quatrième unité à partir de chaque ménage enquêté.

\section{Problématique et hypothèse}

Les observations initiales, effectuées dans les territoires squattés ciblés, nous font supposer que les habitants sont parvenus à surmonter les conditions de vie précaires. Les femmes et les enfants de moins de cinq ans, étant les plus fragiles de ces quartiers ne semblent pas manifester de problèmes de santé inquiétants. Pourtant, la situation détériorée des conditions de vie dont témoigne la précarité de l'habitat, le manque des infrastructures de base et la grande pauvreté des habitants, pourrait aboutir à des problèmes de santé parfois graves. Ceci dit, nous constatons que l'accessibilité aux soins nécessaire réduit l'impact des vulnérabilités sur la santé des habitants des quartiers squattés. Cette accessibilité facilitée est la résultante de l'émergence, dans la ville d'El-Mina, d'acteurs de santé qu'il fallait identifier. Mais d'abord, quel est l'état de santé effectif des femmes et de leurs enfants dans ces quartiers ? Ces femmes adoptentelles des comportements protecteurs vis-à-vis de leur santé et de celles de leurs jeunes enfants, notamment au cours de la phase périnatale? Quelle influence exerce la proximité géographique sur les choix de recours aux fournisseurs de soins? Ces 
derniers ont il créé un réseau de solidarité dans la ville d'El-Mina pour aider les habitants des quartiers squattés dans l'accessibilité aux soins nécessaires?

Par solidarité nous entendons un réseau de soutien aux populations les plus nécessiteuses formé par les acteurs de soins existants dans la ville d'El-Mina et qui met en œuvre des dispositifs favorisant l'aide médicale des pauvres.

Figure 1 : Extrait du questionnaire Alissar ELIAS

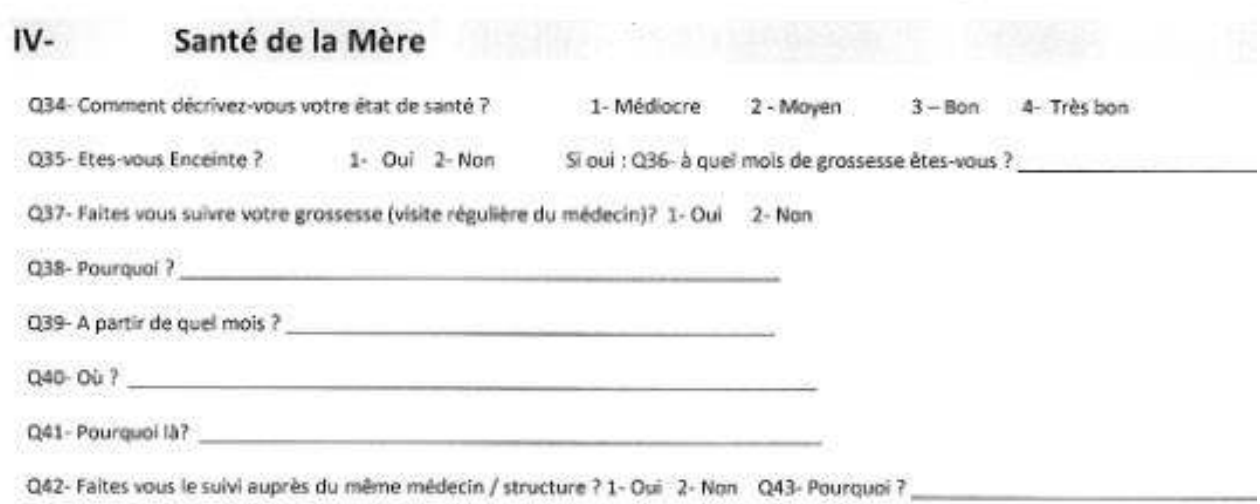

\section{Exploration des choix des destinations aux soins nécessaires et analyse spatiale des données}

21 Des compléments d'enquêtes ont été menés, en 2011 et 2012, auprès des structures de soin existantes dans la ville d'El-Mina. Le but était de comprendre le rôle qu'exercent ces centres dans l'offre de soin disponible et dans la facilitation d'accès aux soins de la population des femmes et des enfants habitant les quartiers squattés sélectionnés. Ces enquêtes ont ciblé les dispensaires, les cabinets libéraux des médecins pédiatres et gynécologues, les organisations non gouvernementales et les associations caritatives locales afin de les localiser et pour apprendre davantage sur leurs programmes de soins mis en place dans la ville. Les personnels de santé rencontrés dans ces derniers ont répondu à des questionnaires différents dont les questions ouvertes sont liées aux services spéciaux offerts aux populations démunies.

Ainsi, 9 dispensaires opérant dans la ville ont été inclus dans l'enquête. Concernant les cabinets privés des spécialistes, les 3 premiers qui figurent parmi les gynécologues et pédiatres les plus visités par les mères et les enfants ont été choisis pour un entretien plus détaillé. De même, les associations locales qui offrent des aides financières pour les soins médicaux aux habitants de la ville ont été visitées et localisées (cartes 3 et 4).

Pour localiser les dispensaires, les cabinets libéraux et les hôpitaux identifiés au cours de l'enquête, nous avons d'abord sollicité la municipalité d'El-Mina. Le département des SIG nous a fourni une carte montrant les numéros des parcelles de toute la ville tout en précisant celui correspondant à chaque centre de soins situé dans la ville. Ces localisations ont été vérifiées lors des visites effectuées auprès des dispensaires et cabinets libéraux visés. Pour les cabinets libéraux et les hôpitaux se trouvant à l'intérieur des limites municipales de la ville de Tripoli, un travail de terrain a été mené afin de préciser la localisation de ces structures se servant du plan de la ville où figurent les noms des rues. Les adresses des cabinets libéraux, notamment les noms des 
rues et des immeubles, ont été trouvés dans un annuaire développé par l'ordre des médecins du Liban nord. Pour situer les associations caritatives, nous avons utilisé les numéros de parcelles fournies par la municipalité d'El-Mina (carte 10).

Les localisations géographiques identifiées ont été analysées sur ArcGIS versions 9.3 et 10, puis transformées en cartes thématiques. Les points représentant les centres de soins fréquentés et les quartiers squattés ciblés forment un réseau de connexité et sont reliés par les voies de circulation routières. Le réseau mis en évidence dans la ville d'ElMina prend la forme d'un graphe dont chaque nœud (qui représente soit un centre de soin, soit un des quartiers squattés ciblés) « admet une liaison avec au moins un autre, il n'existe pas de lieu qui ne soit pas desservi par au moins une liaison (Pumain D., Saint-Julien T, 1997) ». La connexité est définie comme étant « une propriété essentielle des réseaux, qui caractérise leur aptitude à mettre en relation tous les nœuds du territoire qu'ils desservent (idem, p.97.)». La carte du réseau a servi dans le développement d'une matrice des écarts pour calculer le centre de soin le plus accessible. Ces données ont permis le calcul de l' Indice d' Accessibilité se basant sur la méthode de Shimbel «qui permet de donner une mesure normalisée de l'accessibilité d'un sommet dans un graphe (ib idem, p. 110)».

Pour calculer cet indice deux matrices des écarts ont été construites, une intégrant les cabinets libéraux des pédiatres et une autre les cabinets libéraux des gynécologues, à partir de la mesure des longueurs des arêtes séparant chaque deux nœuds (ou sommets) dans le graphe. L'arête est la ligne qui constitue le trajet parcouru à pied par les habitants de chaque quartier (transformé en point central de la surface du quartier) sur les voies principales d'El-Mina et les rues formant le chemin le plus court. Ces arêtes n'étant pas de longueur égale. Concernant les structures de soin situées à l'intérieur des limites municipales de la ville de Tripoli, les arêtes sont les voies principales aboutissant à ces cabinets ou hôpitaux visités par les habitants des quartiers squattés ciblés par l'étude. Les distances obtenues ont été introduites dans la matrice pour pouvoir calculer la somme des écarts (Pumain, Saint-Julien, 1997) et puis identifier le point le plus accessible.

26 L' Indice d'Accessibilité de Shimbel « rapporte la somme des écarts de tous les sommets à la somme des écarts d'un sommet. Le sommet dont l'indice est maximum est celui qui est le plus accessible (Pumain D., Saint-Julien T., 1997, p. 110)».

$\mathrm{SHi}=\{\Sigma \mathrm{i} \Sigma \mathrm{j} \mathrm{E}(\mathrm{i}, \mathrm{j})\} /\{\Sigma \mathrm{iE}(\mathrm{i}, \mathrm{j})\}$

27 Cet indice est utile pour essayer de comprendre l'influence de la distance géographique sur les choix des populations habitant les quartiers squattés étudiés dans le recours aux soins.

\section{Les ressources économiques façonnent l'accessibilité aux soins des populations démunies}

L'accessibilité aux soins des populations pauvres est limitée par le manque des ressources économiques et le manque des infrastructures adéquates. «Au sein des pays à bas et moyen revenue, les pauvres ont moins accès aux services de santé (Peters D. et al, 2008)». Une étude ciblant les pays en voie de développement considère que "l'accessibilité géographique joue un rôle important dans l'accès au soin. Une relation inverse entre la distance et le temps du trajet effectué aux établissements de santé et 
l'utilisation des services de santé a été démontrée comme obstacle important à l'accès (Ann. N.Y, 2008)».

A l'échelle du Liban, le recueil national de la santé affirme que « la couverture sanitaire de la population est inadéquate» et qu'il existe une grande disparité dans la distribution géographique des services offerts «ce qui réduit l'accessibilité de la population à l'utilisation des services de santé (L'Institut de gestion de la santé et de la protection sociale, l'Université Saint-Joseph, 2012)». Or, une étude effectuée en 2012 démontre que l'accessibilité aux soins engage d'autres paramètres que la localisation géographique. Celle-ci montre que les personnes engagées dans un activisme politique ont un meilleur accès aux services de santé que d'autres (Chen et Cammett 2012).

Cependant, les données sur l'accessibilité géographique des populations vulnérables aux soins sont manquantes au Liban; elles ne sont pas disponibles à l'échelle des communes urbaines. Des études descriptives ont été effectuées sur la totalité du territoire libanais ne prenant pas en considération des quartiers dont les déterminants urbains et socio-économiques sont défavorisés. Dans ces études l'aspect géographique a été introduit dans la localisation des problèmes de santé selon les caza sans donner des caractéristiques typologiques aux régions étudiées.

31 Parmi celles-ci, le projet PAPFAM (Pan Arab Project for Family Health) mené en 1996 qui a ciblé un échantillon de 4600 ménages répartis sur la totalité du territoire libanais. Le projet avait comme objectif principal de construire une base de données sur les caractéristiques sanitaires des femmes mariées âgées de moins de 55 ans et les enfants moins de 5 ans. En même temps, l'étude cherchait à évaluer les soins primaires au cours de la grossesse et de l'accouchement (pour les 5 ans précédant l'enquête). Ces informations étaient nécessaires afin de pouvoir préparer les stratégies et programmes pour atteindre l'objectif de la «Santé pour tous » qui était fixé pour l'an 2000.

Une autre étude effectuée par PAPFAM (2004) au Liban a évoqué le niveau éducatif dans l'accès aux soins assumant que les pourcentages des consultations sont aussi plus élevés chez les enfants dont les mères ont atteint un niveau secondaire et postsecondaire. L'éloignement des services de soin n'était pas considéré comme une raison importante, à l'exception des femmes résidant au gouvernorat de la Beqaa. Le prix des consultations et du traitement $(18.3 \%)$ constituaient la troisième raison empêchant le recours au soin. Ces contraintes d'ordre économique et culturel, ont fait que $52.2 \%$ des mères dont les enfants ont souffert de diarrhée n'ont pas consulté un médecin pour les traiter (Arab League, Ministry of Social Affairs (Lebanon), Central Administration of Statistics, 2004).

33 En vue des constatations mises en évidence à l'échelle de tout le pays, nous essayerons de vérifier à l'échelle urbaine l'accessibilité aux soins des populations particulièrement celles habitant les quartiers squattés. Notre étude se base sur une méthodologie principalement orientée par une analyse spatiale de l'offre de soins existant dans la ville d'El-Mina.

\section{Les territoires squattés dans la ville d'El-Mina}

34 De nos jours la ville d'El-Mina est divisée en deux entités urbaines principales, la ville ancienne qui est le centre de la ville et la banlieue. A son tour, la ville ancienne se divise en deux secteurs principaux qui sont Tourab Al-Islam et Tourab Al-Nasara . Les habitants 
de cette partie de la ville comptent 18824 personnes équivalentes à $35 \%$ (Conseil de Développement et de Reconstruction, 2006) de la population totale de la ville. La banlieue englobe les nouveaux quartiers qui se sont développés à partir des années 60 et les quartiers irréguliers qui se situent le long de la côte, vers l'Ouest et le Sud-Ouest $\mathrm{du}$ centre. Ces quartiers rassemblent une population pauvre occupant des logements aléatoires où les infrastructures de base sont inadéquates et souvent inexistantes. Malgré ces conditions de vie défavorisées, ces quartiers demeurent hors des plans d'amélioration des municipalités concernées.

35 Ces territoires (carte 1), partie « la plus cachée des villes » (AGIER M, 1999) sont donc nettement moins bien pourvus en infrastructures de base également à cause des nouveaux COS (Coefficient d'Occupation des Sols) fixés en 2007 par le dernier plan directeur de la ville qui classe la zone côtière en parcelles destinées aux investissements touristiques et résidentiels de luxe. Le quartier le plus touché par cette nouvelle réglementation est Hay El-Tanak (Carte 1), dont les habitants reçoivent des menaces d'expulsion et sont actuellement en conflit juridique avec les propriétaires effectifs des parcelles qu'ils squattent. Les quartiers squattés se caractérisent par l'habitat insalubre, fragile et incertain dévoilant une inégalité d'accès au marché de logement.

36 Faut-il rappeler que le marché foncier au Liban est victime de spéculations ${ }^{3}$ empêchant les populations démunies d'accéder à des logements décents. Ceci est le résultat de la disposition du secteur de l'immobilier par des hommes d'affaires qui le dirigent pour leurs propres intérêts avec l'absence d'une stratégie nationale de logement.

Dans la ville d'El-Mina, les quartiers squattés ne forment pas un tissu urbain continu. Ces territoires, qui ont émergé à des dates différentes dans des endroits séparés de la ville mais pas pour autant très éloignés, côtoient les quartiers réguliers de la ville. Contrairement, par exemple au cas d'Ouagadougou, au Burkina Faso, où le non loti constitue une ceinture continue qui encercle la ville lotie. Une étude effectuée par l'IRD à Ouagadougou, visait également l'estimation des conséquences de l'urbanisation sur la santé des populations tout en distinguant « les zones construites légalement (le loti) ou illégalement (le non loti) ». La stratification de la ville a été effectuée à partir d'une image satellite panchromatique. Le but était de prouver une exposition inégale aux risques sanitaires en préparation de "l'enquête domiciliaire de santé auprès des populations résidant dans les différents types d'espaces urbains (Vallée J., Fournet F. et al. ; 2006 ; p. 394) ». Or, à Ouagadougou, comme dans la ville d'El-Mina, « la progression du non loti est un réel problème dû principalement à la spéculation foncière qui fait rage » (idem).

38 Les trois quartiers squattés, Hay El-Tanak, El Masaken El-Chaabiyah et Haouch, que nous avons sélectionné dans notre étude recouvrent une surface cumulée d'environ $113245 \mathrm{~m}^{2}(11,32 \mathrm{ha})^{4}$ et abritaient en 2009, 665 ménages $^{5}$. Bien que tous squattés, chacun de ces espaces possède une histoire différente qui a abouti à un accroissement, une morphologie (carte 1), et des conditions de vie quelques peu différentes. Le quartier de Haouch qui se situe au Nord des deux autres, est le plus restreint en surface regroupant des bâtiments accolés les uns aux autres, qui se développent en hauteur. Par contre, Hay El-Tanak semble plus étendu en s'étalant horizontalement et où l'habitat est éparpillé comme le montre la carte 1. Cependant, El-Masaken El-Chaabiyah est un territoire plus organisé prenant la forme d'un lotissement. 
Les habitants de ces territoires souffrent de plusieurs aléas liés à l'insécurité et l'exiguïté du logement, au manque d'infrastructures sanitaires de base qui atteignent un seuil critique grave. Dans cette partie de la ville les impacts des conditions de vie sur la santé n'ont pas encore été explorés. Ci-après une description plus détaillée des territoires ciblés décrivant leur genèse et développement urbain.

Carte 1 : Quartiers squattés ciblés de la ville d'El-Mina

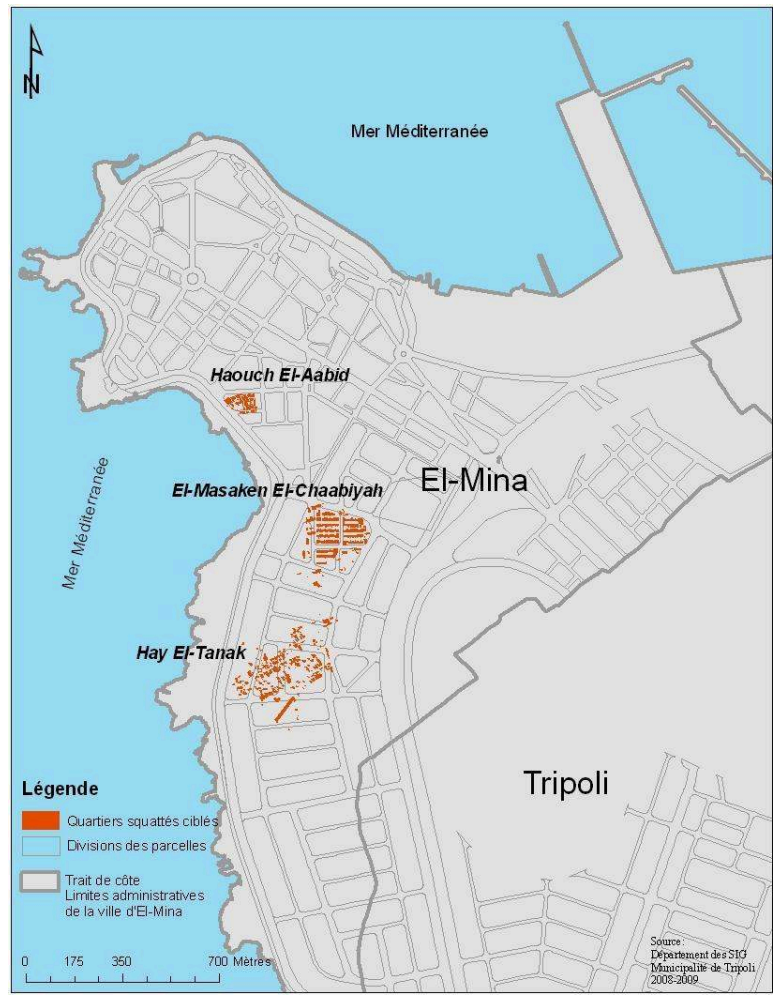

\section{Hay El-Tanak}

40 Le quartier se trouve dans un secteur de la ville appelé Bassatine ${ }^{6}$ d'El-Mina en référence aux orangeraies et aux champs de roseaux. Implanté sur un terrain en cuvette vacant, à quelques dizaines de mètres en bordure du rivage (carte 1), plusieurs futurs émigrés y campaient avant d'embarquer clandestinement sur le premier cargo quittant le port de Tripoli. Le premier baraquement y a été édifié en 1940. Mais avec l'accentuation de l'exode rural les paysans se sont installés illégalement après avoir été infiltrés illicitement dans des champs puisqu'ils ne possèdent les moyens pour louer des logements dans les quartiers du centre. Les baraques qui composent ce quartier irrégulier, sont construites avec du parpaing et du béton (photo 1) surmontés de toits formés de la tôle (photo 2) et des planches d'amiante. Le quartier s'est fortement densifié au cours de la guerre civile accueillant de nouvelles populations (1975-1990). La population y est à majorité libanaise. D'après notre enquête, le quartier rassemble 963 habitants occupant 185 unités d'habitation ${ }^{7}$. A Hay El-Tanak, le plus démuni des trois territoires, le réseau municipal et la borne-fontaine sont les deux sources primordiales d'eau domestique. Il s'agit en fait de canalisations manuellement implantées et dérivées clandestinement à partir du réseau d'eau public passant près du 
quartier branché aux immeubles réguliers construits dans le voisinage. Dans ce secteur, les fosses traditionnelles sont le moyen premier d'évacuation des eaux usées.

Photo 1 - forme de l'habitat à Hay El-Tanak

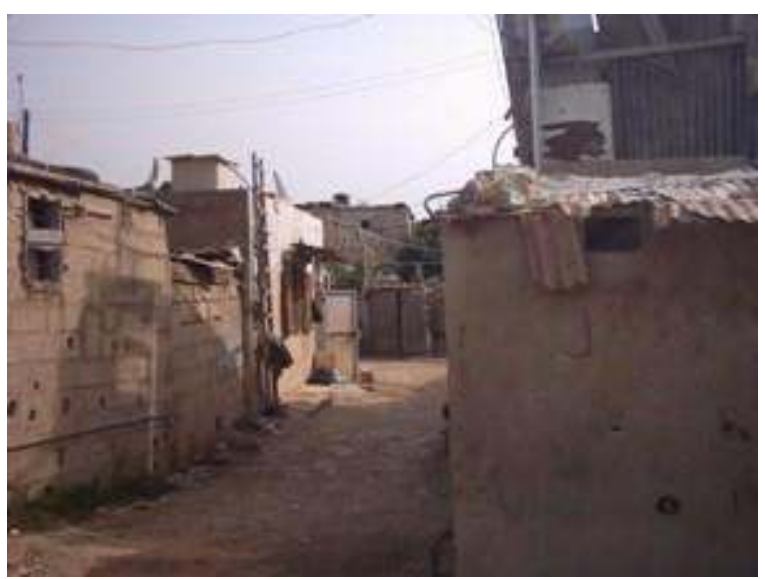

Source : Elias A, 2009.

Photo 2 - un toit en tôle fortifié par des planches de bois à Hay El-Tanak

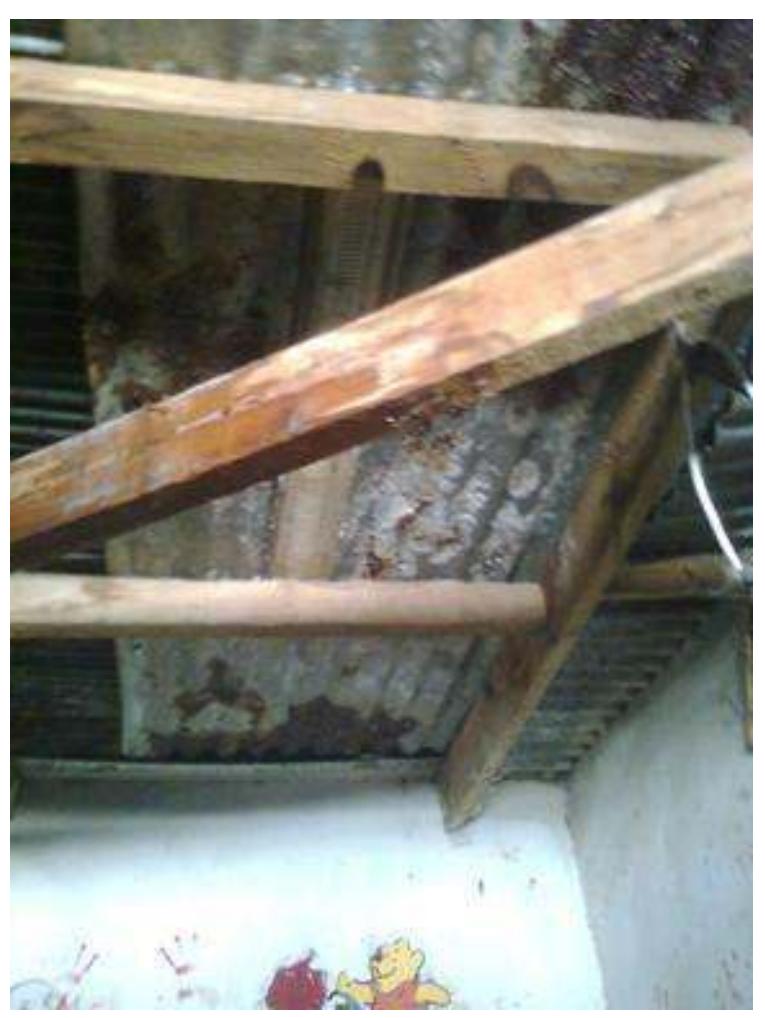

Source : Elias A, 2009. 
Photo 3 - déchets solides collectés à Hay El-Tanak

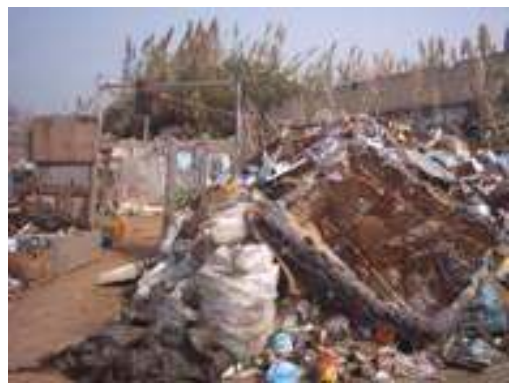

Source : Elias A, 2009.

\section{Haouch}

41 C'est un des quartiers les plus anciens de la ville d'El-Mina. Il s'est développé au début du $19^{\text {ème }}$ siècle. Les premiers habitants venaient d'Afrique : Maroc, Soudan et Sénégal. Plusieurs anecdotes expliquent l'arrivée de ces populations et leur installation sur ces terrains. Pour certains ces populations ont été amenées par un homme d'affaires local, pour travailler dans la ville. D'autres racontent que ces ouvriers étaient à bord d'un bateau qui a coulé au bord de la côte. Ainsi, ils ont été sauvés du naufrage et se sont installés près de la rive. Ces premiers arrivants travaillaient comme des ouvriers journaliers dans la Municipalité, dans le charriage des produits dans les douanes du Port de Tripoli et dans la pêche. Au début, les parcelles sur lesquelles est édifié le quartier appartenaient à une grande famille politique de Tripoli qui a fait installer ces ouvriers étrangers sans aucune réclamation de payement. Mais avec le temps, les habitations se sont étendues et les murs des baraques initiales (en bois et tôle) se sont «durcis " en parpaing et béton (photo 4). D'après un précédent président de la Municipalité, $80 \%$ des habitations sont construites sur des terrains privés. Actuellement, ce quartier regroupe 425 habitants occupant 94 unités de logement ${ }^{8}$. A Haouch les puits privés et les puits communs sont utilisés comme source d'eau alors que le réseau d'égout sert pour se débarrasser des eaux usées. 


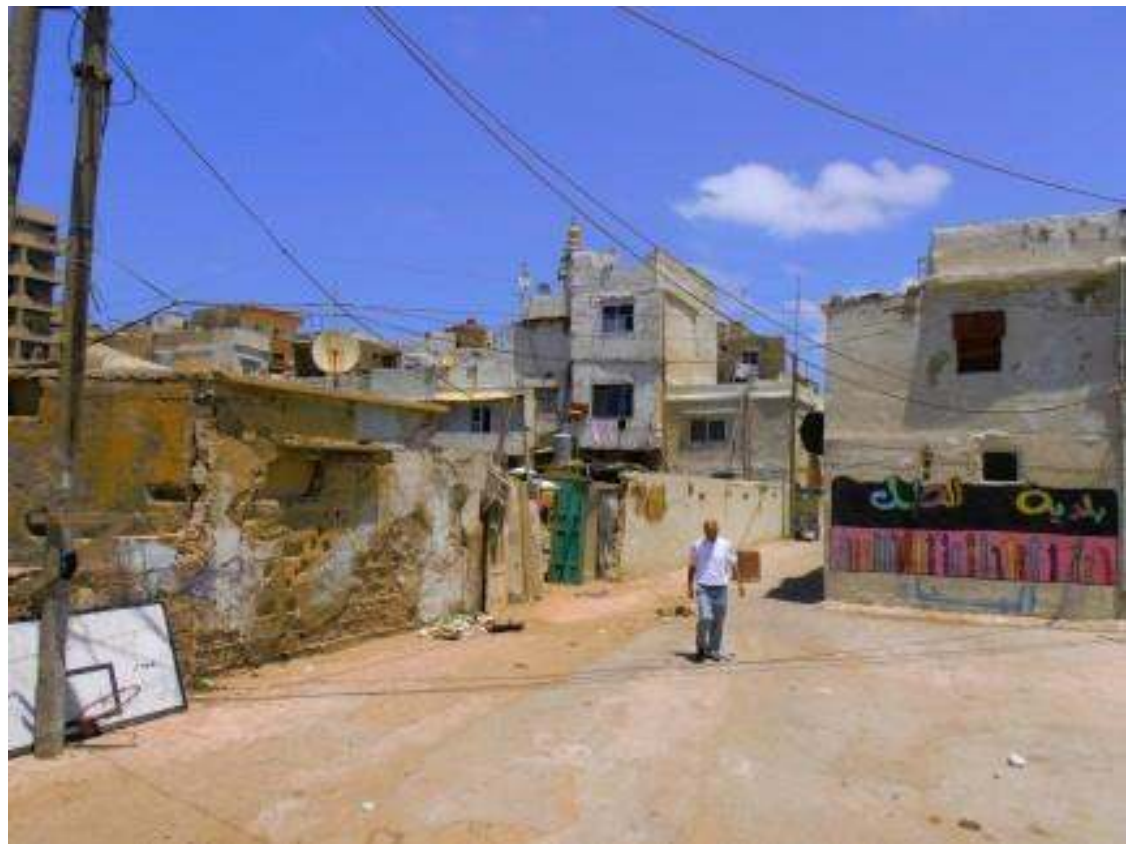

Source : Elias A, 2012.

\section{El Masaken El-Chaabiyah}

42 Au début des années 1970 le quartier a été loti dans le cadre d'un plan adopté par le ministère des affaires sociales et le ministère de logement et des coopératives. Le plan prévoyait la construction de logements sociaux consacrés aux ménages à bas revenus. Ce lotissement de 200 unités a été planifié dans la ville d'El-Mina. Les travaux ont débuté en 1971, mais bien que les maisons aient été édifiées et les rues tracées, le déclenchement de la guerre de 1975 a suspendu l'exécution du projet. Les premiers déplacés de la guerre, issus de diverses régions, ont squatté les logements et ont euxmêmes achevé les travaux de construction.

Les unités résidentielles étaient initialement composées de deux étages (photo 5). Chaque appartement comprenait une cuisine, une toilette et une salle de bain, un salon et 2 ou 3 chambres à coucher. Au niveau du rez-de-chaussée, les appartements disposaient d'un espace qui pouvait être transformé en petit jardin. Les ruelles qui séparent les habitations sont cimentées (photo 6) et les habitations sont connectées aux infrastructures d'assainissement. Les habitants utilisent le réseau municipal et les puits privés pour l'usage domestique. Pour évacuer les eaux usées ces lotissements sont branchés au système d'égout de la ville.

Ce quartier à $90 \%$ planifié a connu une forte densification au courant des années à cause de l'arrivée d'une large population de nationalités différentes, tout en attirant des magasins et des industries qui s'y sont installées. Les ménages s'étant élargis, les résidents ont dû diviser les logements afin d'y loger leurs enfants mariés, incapables de trouver un logement abordable en ville. Certaines familles ont ajouté des pièces ou des étages supplémentaires sur les toits. 
Photo 5 - forme de l'habitat aux Masakens

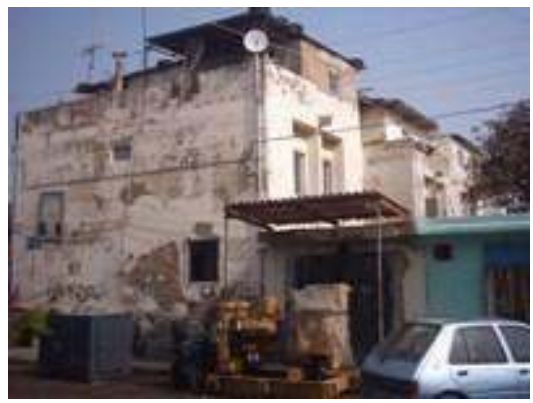

Source : Elias A, 2009

Photo 6 - rue interne entre les lotissements aux Masakens

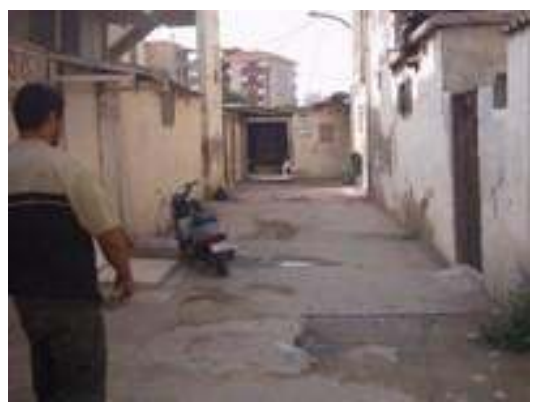

Source : Elias A, 2009

Ce qui suit surligne l'origine des populations occupant les quartiers squattés ciblés.

\section{Une population à majorité libanaise}

Dans le quartier d' El Masaken El-Chaabiyah un pourcentage élevé des habitants est originaire de la ville d'El-Mina. Une autre majorité est née dans le quartier alors qu'une proportion est issue de la ville de Tripoli et de la région de Akkar (Gouvernorat proche de Tripoli). A Hay El-Tanak les personnes rencontrées sont principalement originaires de la Syrie parmi lesquelles un grand nombre a reçu la nationalité libanaise dans les années 90. La seconde grande partie de la population est issue de la ville d'El-Mina ou est née dans le quartier. Quelques personnes sont même venues de la région de Akkar ou du quartier d' El Masaken El-Chaabiyah . Par contre, à Haouch la tranche importante dérive de la ville d'El-Mina, est née dans le quartier ou est provenue de la capitale Beyrouth au cours des années de la guerre civile déclenchée en 1975. La grande majorité de ces populations survivent avec des revenus mensuels très bas ce qui aggraverait aussi l'accessibilité aux soins nécessaires.

\section{Ménages dont le statut économique est defavorisé}

47 A Hay El-Tanak dont les chefs de ménages travaillent surtout dans la pêche et la collecte des déchets, plus de la moitié des ménages (51\%) survivent avec moins de 600 milles L.L. par mois (équivalent à 354 Euros). Une grande proportion aussi (36\%) touchent 300 milles L.L. (177 Euros) et moins par mois. Il en est de même pour le quartier de Haouch où $44 \%$ des ménages vivent avec un revenu mensuel variant entre 300 et 600 milles L.L. 
Pourtant $30 \%$ des ménages se contentent d'un salaire mensuel total égal ou inférieur à 300 milles L.L. et $22 \%$ bénéficient d'un montant mensuel situé entre 600 milles L.L. et 900 milles L.L. Dans le quartier d' El Masaken El-Chaabiyah le pourcentage le plus élevé (44\%) des ménages saisissent entre 300 milles et 600 milles L.L. mensuels. Avant 2008, le salaire minimum fixé par l'Etat Libanais était égal à 300 milles L.L.; il est devenu 500 milles L.L. (295 Euros) après cette date. En 2012, le seuil minimum a été fixé à 675 milles L.L. (398 Euros) par mois. Ces chiffres signifient que ces populations disposent de peu de ressources financières pour survivre sachant que la taille moyenne des ménages dans ces quartiers est de 5.3 personnes (légèrement supérieure à la moyenne nationale qui est 4.23 personnes).

Partant du fait que nos cibles principales sont les femmes en âge de reproduction, il s'est avéré indispensable d'explorer leur niveau éducatif.

\section{Des femmes faiblement instruites}

49 Les femmes enquêtées, qui sont des mères de familles dans la plupart des cas étudiés, n'ont pas dépassé les classes primaires (9.6\% à Hay El-Tanak, $8 \%$ à Haouch et $8.2 \%$ au quartier d' El Masaken El-Chaabiyah ). La majorité a quitté l'école entre l'âge de 11 et 12 ans. D'autres n'ont pas eu la chance d'entrer à l'école qui était perçue comme inutile pour les filles dont le seul sort était le mariage. Un pourcentage minime a eu la chance d'arriver au brevet (moins de $1 \%$ à Hay El-Tanak et $1.1 \%$ au quartier d'El-Masaken ElChaabiyah).

Victimes de conditions économiques détériorées, et de croyances culturelles et parfois religieuses hyper protectrices, ces femmes n'ont pas pu jouir de leur droit à l'éducation. Par ailleurs, ces femmes n'exercent pas d'activités économiques et passent la plupart de leurs temps à l'intérieur de l'unité résidentielle s'occupant de la famille et des affaires ménagères. Or, à l'échelle nationale, PAPFAM (2004) rapporte que plus le niveau éducatif de la mère est élevé plus le soin maternelle au cours de la grossesse est respecté. La prévalence du suivi prénatal est de $80 \%$ chez les femmes illettrées et $100 \%$ chez les femmes universitaires.

51 Ainsi, vu la situation financière détériorée et le niveau éducatif bas des femmes ciblées, comment celles-ci et leurs enfants arrivent à accéder aux soins? Compte tenu des résultats obtenus à l'échelle nationale qui montrent que le soin au cours de la grossesse est plus élevé chez les femmes universitaires, quelle pourrait être la situation dans les quartiers squattés de la ville d'El-Mina?

Dans les parties qui suivront nous présenterons les résultats relatifs à la santé des femmes et des enfants de moins de cinq ans habitant les territoires squattés de la ville d'El-Mina. Nous soulignerons les comportements adoptés lors du recours aux soins nécessaires durant la phase périnatale et quand des symptômes de diarrhée, de fièvre et de toux apparaissent chez les enfants.

\section{Suivi élevé de la grossesse dans les quartiers squattés d'El-Mina}

La grossesse est divisée en trois trimestres, pourtant la période périnatale inclut un quatrième trimestre formé des trois mois qui suivent l'accouchement. En effet, «la 
période anténatale est sans aucun doute la phase cruciale de tout le cycle périnatal pour le développement d'un enfant sain » (Schult D. ; 1978 ; p. 101). Une fois enceinte, la visite régulière auprès du médecin est nécessaire pour savoir si la femme souffre d'affections qui peuvent générer des complications et ainsi affecter sa santé, celle de l'embryon et du fœtus. Selon les normes fixées par l'OMS, adoptées aussi au Liban, une femme enceinte doit consulter le médecin au moins quatre fois au cours de sa grossesse. Les indicateurs publiés par l'OMS montrent que la plupart des femmes au Liban reçoivent des soins prénataux (96\%) par des personnels qualifiés. Or, ces chiffres sont amalgamés et ne réfléchissent pas les disparités spatiales.

A l'échelle des territoires squattés, nos enquêtes ont démontré que $65 \%$ des femmes font suivre régulièrement leur grossesse à partir du premier mois de gestation. Bien qu'inférieur au chiffre national, ce pourcentage nous fait remarquer qu'une grande proportion des femmes habitant les quartiers squattés ont accès aux soins prénataux malgré la situation socio-économique défavorisée. Répartis sur les 3 quartiers squattés, ces pourcentages sont les plus élevés dans le quartier d' El Masaken El-Chaabiyah (77 \%), suivi par Haouch (61\%) puis par Hay El-Tanak (56\%), comme le montre la carte 2.

Carte 2 - Suivi de grossesse déclaré par les femmes habitant les quartiers squattés ciblés

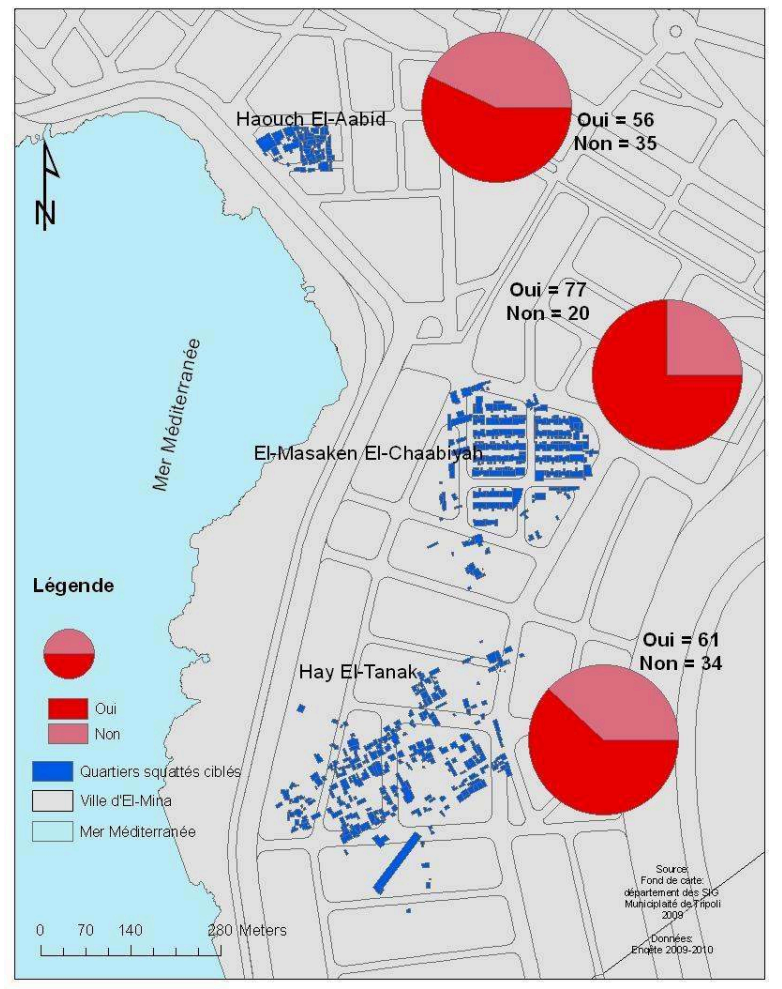

Source : Elias A, 2009-2010.

Les suivis réguliers de la grossesse se reflètent par des taux d'accouchements compliqués ne dépassant pas $4,1 \%$ des cas enquêtés comme le montre le graphique 1 . Les accouchements compliqués forment un indicateur de morbidité au cours de la phase périnatale, menaçant la vie de la mère et de l'enfant en même temps. Dans les zones enquêtées, les cas les plus déclarés sont les césariennes, alors que certaines femmes ont révélé avoir eu des accouchements compliqués sans préciser le type. Le taux des césariennes est le plus élevé à Hay El-Tanak (6.3\%) alors que les accouchements 
compliqués d'autres types sont les plus remarqués chez les femmes habitant aux Masakens $4.1 \%$. Les deux taux cumulés, révèlent des valeurs presque égales dans ces deux quartiers, 8 \% à Hay El-Tanak et $7.7 \%$ aux Masakens (graphique 1). Ces valeurs sont probablement relatives aux femmes qui ne suivent pas régulièrement leurs grossesses sachant que le pourcentage de suivi de grossesse déclaré est relativement élevé (carte 2) mais n'est pas perçu chez la totalité des femmes interrogées.

56 Le taux des naissances prématurées moyennes varie entre 1,4\% à Hay El-Tanak et 2,7 \% (graphique 1) au quartier d'El Masaken El-Chaabiyah, inférieur aux taux national équivalent à $9 \%$ en 2000 . L'accouchement prématuré ${ }^{9}$ est l'une des causes principales de la mortalité néonatale des enfants.

57 En effet, le suivi de la grossesse pourrait éviter les naissances prématurées qui se divisent en prématurité moyenne (entre 32 et 37 semaines d'aménorrhée) et grande (moins de 32 semaines d'aménorrhée). Dans les 3 quartiers étudiés, la grande majorité des femmes qui suivaient leurs grossesses n'ont pas eu des naissances prématurées.

Pareillement, la majorité des femmes qui suivent leur grossesse n'ont pas déclaré des décès néonataux ${ }^{10}$. Parmi celles qui ne consultent pas un gynécologue durant la grossesse, 3 cas de décès de ce type ont été déclarés à Hay El-Tanak, 1 à Haouch et 1 dans le quartier d'El-Masaken. Le taux de mortalité après la première semaine de vie et le taux de mortalité néonatale précoce atteint chacun 4,9\% à Hay El-Tanak mais ne dépasse pas 0,9 et 0,4\% au quartier d' El Masaken El-Chaabiyah. Ces taux sont les plus aperçus à Hay El-Tanak qui est le plus démuni parmi les trois quartiers squattés. Ce territoire est le plus éloigné du centre de la ville où les structures de soins sont concentrées (carte 4). Ce taux est possiblement indicateur d'une difficulté du recours au soin ou de la vétusté de l'habitat à Hay El-Tanak qui pourrait être nuisible à la santé des nouveau-nés.

\section{Graphique 1 - Les taux relatifs à la santé périnatale à l'échelle des trois quartiers}

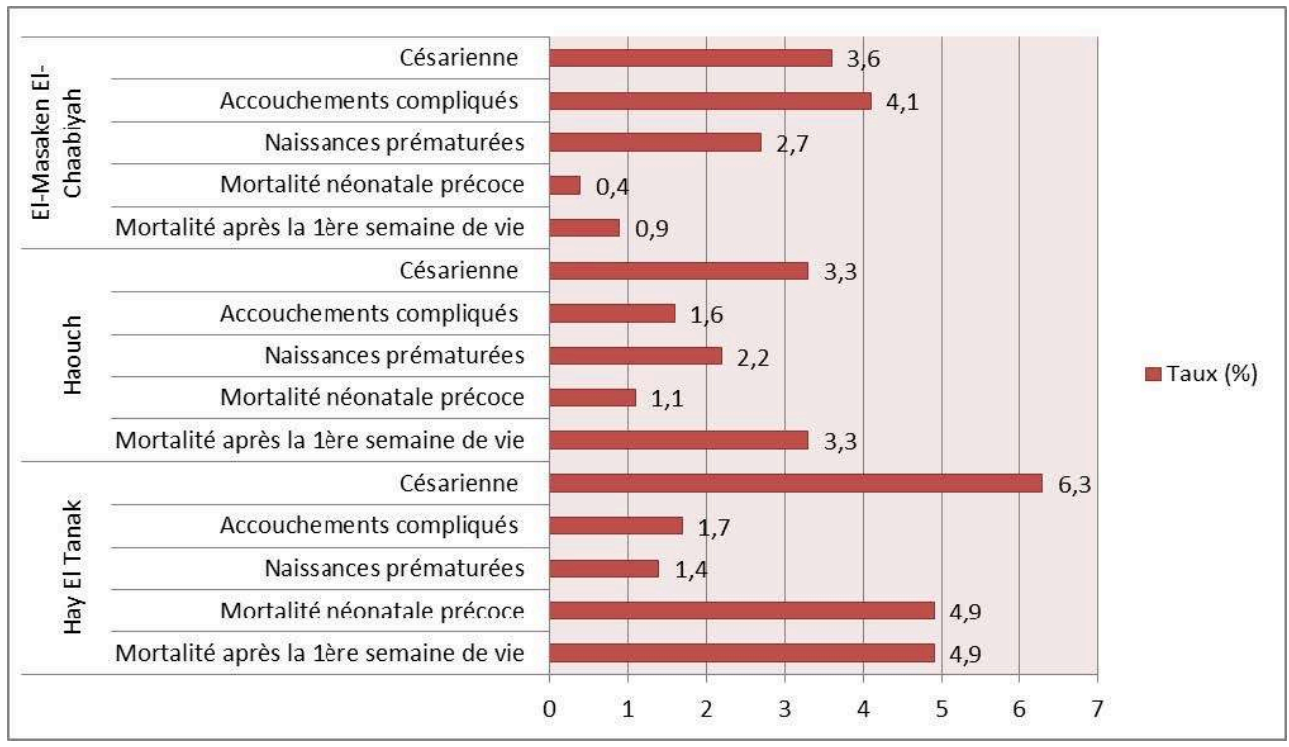

59 A l'échelle nationale, les études ont montré que les mortalités des enfants à un âge inférieur à 1 mois sont dues principalement aux malformations, aux naissances prématurées et aux accouchements compliqués au cours de la première semaine de vie. Cependant, les enfants qui meurent dans les 3 dernières semaines du premier mois de 
vie sont soit infectés par des pneumonies fatales et (ou) sont nés avec un poids très faible. Dans certains cas, ces décès sont dus à des malformations.

Ainsi, nous constatons que, dans les quartiers squattés de la ville d'El-Mina, le suivi de la grossesse abouti à la protection de la femme et de l'enfant au cours de la phase périnatale. Pourtant les moyens financiers et les ressources dont disposent ces populations sont très limitées.

61 Comme le montre le tableau 2, la plupart de ces populations ne sont pas motorisées et se déplacent à pieds $(27 \%)$ ou en utilisant les taxis services ( $38 \%$ transports en commun).

La quasi-totalité ( $98 \%$ ) des ménages ne sont pas couverts par la Caisse Nationale de Sécurité Sociale (CNSS) et $73 \%$ de ceux-ci vivent avec un revenu mensuel inférieur à 600,000 L.L. (354 Euros) (sachant que le seuil minimum a été fixé à 675,000 L.L. = 398 Euros par mois, en 2012). Cependant, dans les trois quartiers les pourcentages de suivi de la grossesse dépassent la moitié des femmes rencontrées.

63 Ceci signifierait des dépenses de déplacement pour accéder aux soins périnataux surtout pour les femmes enceintes habitant les quartiers les plus éloignés des centres de santé, comme Hay El-Tanak . Compte tenu de cette situation financière démunie, les recours aux soins dans ces quartiers pourraient être dérivés de l'existence d'un système de prestation facilitant l'accès aux soins médicaux. Sans pour autant négliger l'effet des politiques de protection maternelle et infantile implantées au Liban depuis l'an 2000 dans le cadre des articles 4 et 5 des objectifs du Millénaire pour le développement lancés par le PNUD. Le faible niveau d'instruction ne semble pas inhiber le recours aux soins durant cette phase dont les mères semblent être conscientes de son importance.

Tableau 2 - Juxtaposition des indicateurs économiques au suivi de la grossesse

\begin{tabular}{|c|c|c|c|c|c|c|c|c|c|}
\hline \multirow[b]{2}{*}{ Quartier } & \multicolumn{3}{|c|}{ Moyens de transport } & \multicolumn{3}{|c|}{ Revenu } & \multicolumn{2}{|c|}{ CNSS } & \multirow{2}{*}{$\begin{array}{c}\begin{array}{l}\text { Suivi de } \\
\text { grossesse }\end{array} \\
\begin{array}{c}\text { Oui } \\
(\%)\end{array} \\
\end{array}$} \\
\hline & $\begin{array}{l}\text { Transport } \\
\text { en } \\
\text { commun } \\
(\%)\end{array}$ & $\begin{array}{l}\text { A } \\
\text { pieds } \\
(\%)\end{array}$ & $\begin{array}{l}\text { Motorisé } \\
\text { (\%) }\end{array}$ & $\begin{array}{l}\text { Inférieur } \\
\text { ou égale à } \\
300,000 \\
\text { L.L. }(\%)\end{array}$ & $\begin{array}{l}{[301,000-} \\
800,000] \\
\text { L.L. }(\%)\end{array}$ & \begin{tabular}{|l|} 
Plus que \\
600.000 \\
L.L. (\%)
\end{tabular} & $\begin{array}{l}\text { Oui } \\
\text { (\%) }\end{array}$ & $\begin{array}{l}\text { Non } \\
\text { (\%) }\end{array}$ & \\
\hline Hay El-Tanak & 34 & 28 & 38 & 38 & 49 & 13 & 6 & 84 & 61 \\
\hline Haouch & 48 & 30 & 22 & 30 & 43 & 45 & 0 & 100 & 56 \\
\hline $\begin{array}{l}\text { El Masaken } \\
\text { El-Chaabiyah }\end{array}$ & 32 & 22 & 46 & 12 & 45 & 35 & 0 & 100 & 77 \\
\hline Total & 38 & 27 & 35 & 27 & 46 & 31 & 2 & 98 & 65 \\
\hline
\end{tabular}

Ce comportement protecteur de la santé maternelle et infantile indique une facilitation de l'accès aux centres de soin, mais quels sont les facteurs qui entrent en jeu?

\section{Recours restreints des enfants aux soins nécessaires}

Concernant la santé des enfants après la phase périnatale et jusqu'à l'âge de 5 ans, les retards dans le calendrier des vaccins obligatoires signifient des recours restreints aux centres de soins. En consultant le graphique 2 nous remarquons que parmi les 111 enfants de moins de cinq ans résidant dans les territoires squattés ciblés, $43 \%$ n'ont pas complété le calendrier des vaccins recommandés à leur âge. Par conséquent, ces enfants se trouvent en risque de contracter des maladies contre lesquelles ils ne sont pas protégés. Des efforts nationaux ont été effectués par le MSP, UNICEF et OMS afin de 
maximiser le taux de vaccination des enfants de moins de cinq ans à travers les campagnes régionales de vaccination.

La dernière campagne nationale anti-rougeole et anti-rubéole a eu lieu en 2008, celle-ci ciblait les établissements scolaires et les crèches. Mais des campagnes précédentes contre la rougeole et la rubéole ont été exécutées avec le soutien de l'OMS en 2001. Une campagne contre la poliomyélite avait eu lieu aussi en 2003. Et suite à la guerre de 2006 l'UNICEF a lancé une autre campagne de vaccination antipolio ${ }^{11}$ dont l'objectif était la protection de 320000 enfants touchés par le conflit. Le calendrier de vaccins est révisé en permanence par le MSP auquel sont ajoutés de nouveaux vaccins.

Mais il faut rappeler que le programme national de vaccination mis en place par le ministère de la santé publique ne couvre pas tous les vaccins indispensables. Ainsi, les parents sont obligés de se rendre chez un pédiatre pour compléter les vaccins non couverts. Ces derniers coûtent le plus cher et ne sont pas disponibles aux dispensaires. Parmi ces derniers la typhoïde $(100,000$ L.L. $=59$ Euros $)$, l'hépatite A, la varicelle $(100,000$ L.L. $=59$ Euro $)$, le rota virus $(150,000$ L.L. $=88$ Euro $)$, et les méningites . Pourtant, les populations des quartiers squattés ne peuvent pas aborder ces prix, donc ils renoncent à suivre les vaccins restants. Ceci signifie des inégalités d'accès aux vaccins protecteurs.

Graphique 2 -Prévalence des symptômes perçus chez les enfants de moins de 5 ans

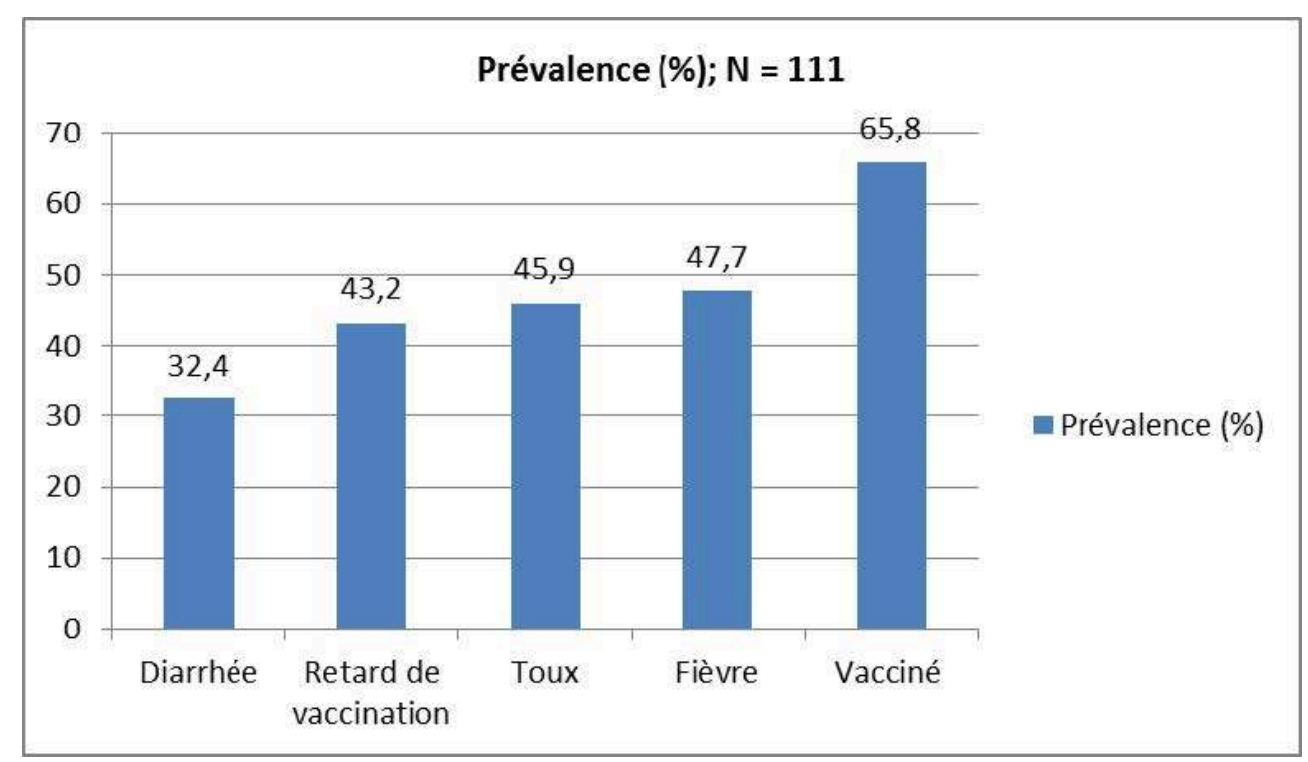

Source : Elias A.

Concernant les autres symptômes étudiés, l'enquête a montré que $32 \%$ des enfants souffraient de diarrhée, $45 \%$ de toux et $47 \%$ de fièvre au cours des 15 jours précédant notre enquête (graphique 2). Les fièvres chez les enfants de cet âge sont généralement provoquées par des infections respiratoires, des diarrhées ou des maladies hydriques transmissibles. De même, la diarrhée peut provoquer la déshydratation si elle dure plusieurs jours, ce qui peut aboutir à la mort de l'enfant privé des sels minéraux nécessaires à la vie. Celles-ci sont dues à une contamination de l'eau ou des aliments par des bactéries, des virus et des parasites particulièrement dans les milieux où il $\mathrm{y} a$ un manque d'hygiène qui est le cas dans les quartiers squattés ciblés. Les fièvres et les toux peuvent être des indicateurs d'infections respiratoires. Les impacts de l'habitat 
spontané varient avec les saisons puisqu'en hiver «les chambres humides et froides sont envahies par les rhumes, angines, bronchites, grippes saisonnières, bronchopneumonies ", alors qu'en été la chaleur élevée et la mal-ventilation provoquent «les gastrites, colites, entérites, dysenteries et typhoïdes (Semaines sociales du Liban, 1961, p. 36)».

69 Face à ces symptômes, les mères ont été interrogées sur leurs comportements pour y remédier, en répondant aux deux questions suivantes: "Qu'avez-vous fait ?" et "où êtesvous allées?". C'est ainsi que nous avons pu identifier les centres de soins fréquentés par la population étudiée. Il est important de souligner que les mères optent pour l'automédication comme premier choix de remède. Alors nous remarquons que le recours au soin dans la phase prénatale est plus élevé que lorsque les enfants tombent malades.

70 Pour appréhender la situation actuelle, nous procéderons à la présentation du système de santé du Liban et des mécanismes de fonctionnement du système de soin mis en place dans la ville d'El-Mina. Les choix de recours aux soins seront ensuite analysés à la lumière des résultats obtenus suite au calcul de l'indice d'accessibilité aux structures de soins existantes dans la ville.

\section{Centres de soins présents dans la ville d'El-Mina}

71 Les structures principales fournissant le soin au Liban sont les hôpitaux, les cabinets privés et les dispensaires. Les hôpitaux se divisent en privés et publics alors que les dispensaires, qui incluent aussi les centres de soin primaires, appartiennent à l'Etat, à des organisations non gouvernementales, à des partis politiques, à des associations religieuses ou à des individus. Les établissements appartenant à l'Etat sont reliés aux Ministères de la Santé Publique, des Affaires Sociales (MAS) ou de l'Intérieur et des Municipalités.

72 Les fonctions remplies par chacune de ces structures se chevauchent parfois, sachant que les dispensaires sont les plus présents dans les espaces urbains surtout. Les hôpitaux privés sont les plus nombreux au Liban. En 2012, le « réseau hospitalier comprenait 164 institutions publiques et privées réparties sur l'ensemble du territoire libanais et couvrant l'ensemble des spécialités médicales et chirurgicales (Ministère de la Santé Publique, 2012)». Ce réseau inclus 28 hôpitaux publics (dont un avec le statut d'hôpital universitaire) représentant un total de 2550 lits.

73 Le Ministère de la Santé Publique (MSP) affirme que les centres de soin primaire ont joué un rôle dans l'amélioration des conditions sanitaires des populations suite aux programmes préventifs et curatifs implantés dans toutes les régions libanaises. Ces centres couvrent $23 \%{ }^{12}$ des consultations sanitaires par année, à l'échelle du Liban. A Tripoli 7 centres de soin primaire ont été créés jusqu'en 2009, dont 1 centre dans la ville d'El-Mina dirigé par une association religieuse locale, présente dans d'autres régions du Liban Nord. Se basant sur les publications du MSP, le nombre des dispensaires au Liban a dépassé $900^{13}$.

74 Le Ministère envisage l'intégration des dispensaires qui fournissent de bons services dans la chaîne des centres de soins primaires (CSP). Les CSP (photo 7) rendent des services «de suivi pré et postnatal, d'éducation pour la santé, l'usage des moyens contraceptifs, le dépistage du cancer mammaire et cervical et la prise en charge 
thérapeutique et des maladies sexuellement transmissibles (Ducruet J., s.j. 2009) » dans le cadre d'un programme national visant l'amélioration de la santé reproductive " qui fut entrepris au Liban en $1997^{14}$ " par le MSP et le MAS soutenus par les Fonds des Nations-Unies pour la population. Ces structures exécutent aussi le programme national de vaccination (photos 8 et 9) qui a commencé en 1987 avec le soutien du MSP, OMS et UNICEF.

Photo 7 - Centre de soin primaire - soin de la mère et de l'enfant - liste des services offerts

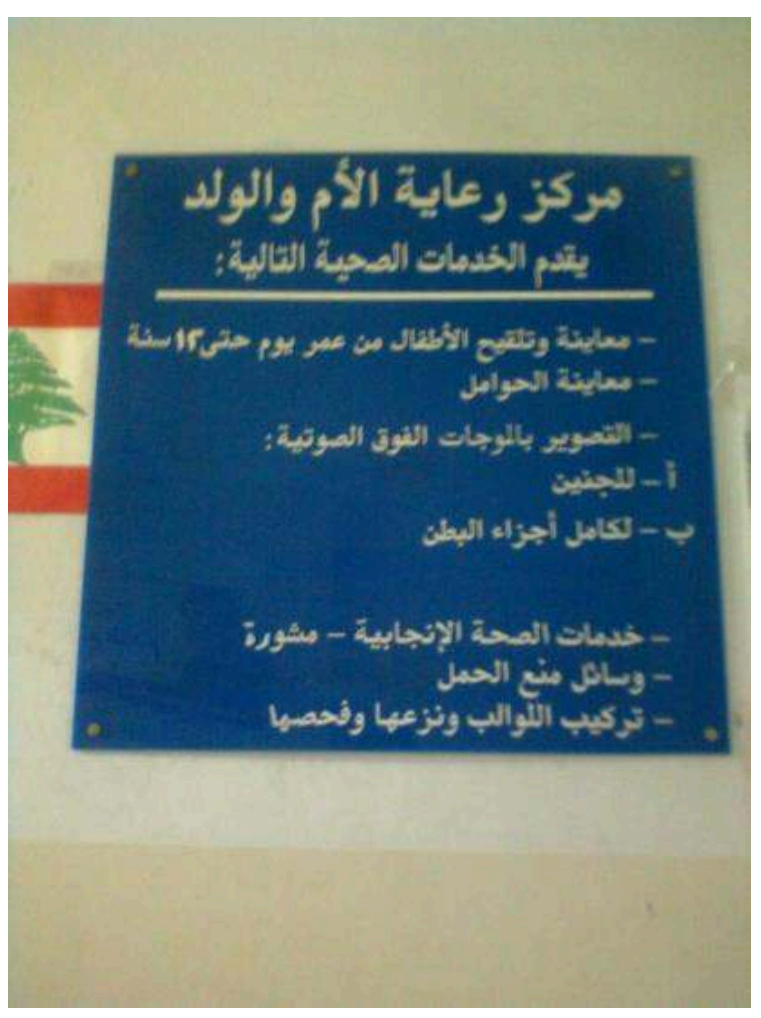


Photo 8 - Calendrier de vaccination

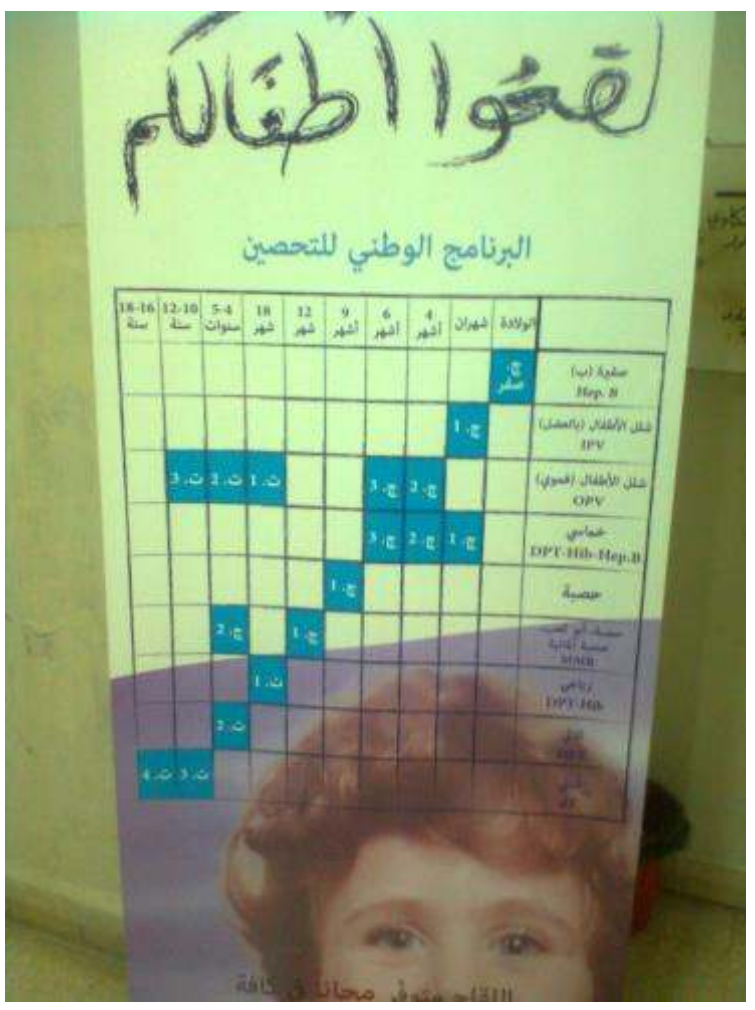

\section{Photo 9 - Brochures sur la vaccination}

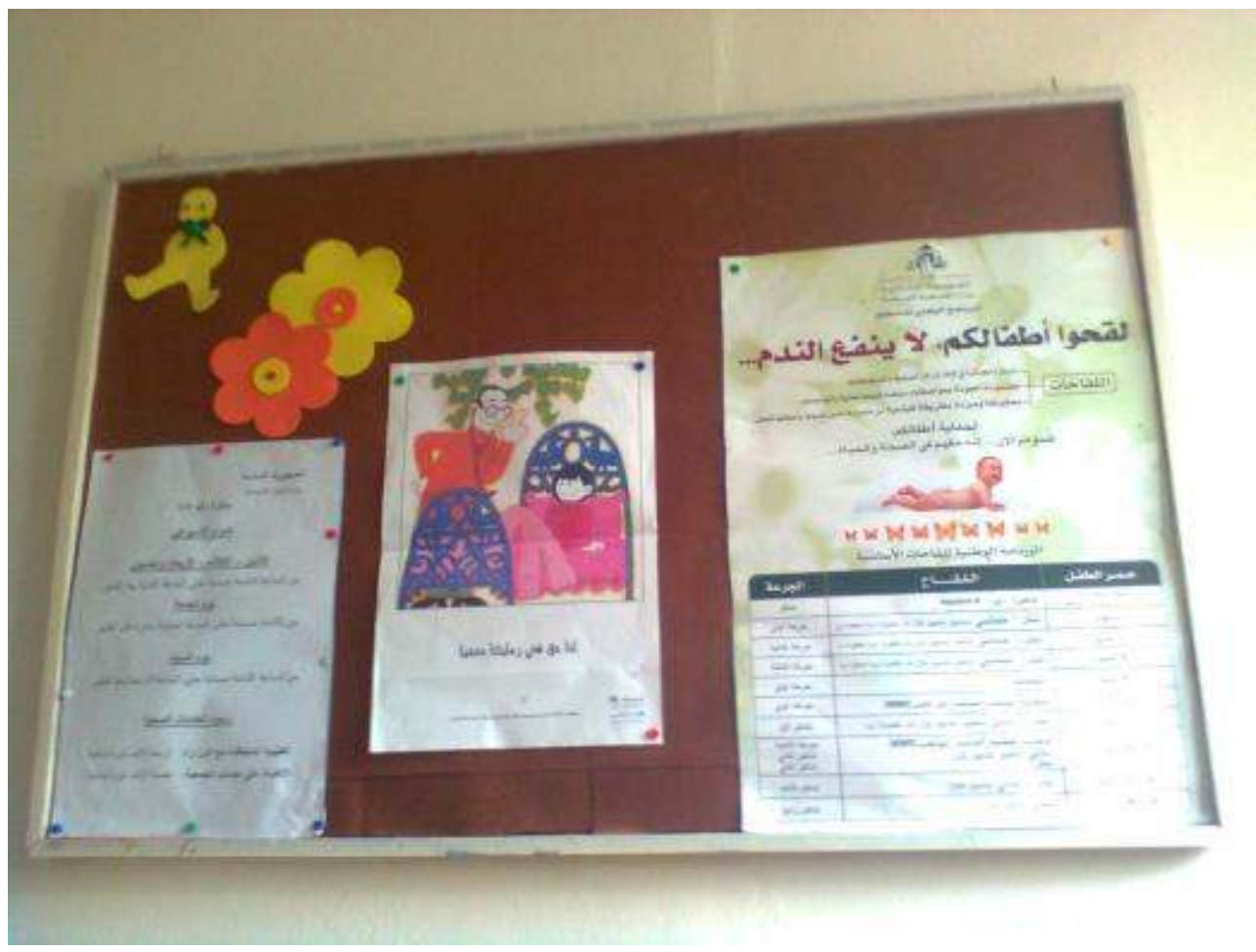

75 La ville d'El-Mina dispose de dix dispensaires ${ }^{15}$, d'un centre de soins primaires (carte 3), de huit cliniques ${ }^{16}$ de pédiatres, de deux cliniques de gynécologues. Pourtant, il y a une vingtaine d'années, la ville était dotée de deux hôpitaux (Housseini et Dar El 
Tawleed). Selon la carte 3, les dispensaires situés dans la ville d'El-Mina sont dirigés par des ONG comme Al-Najdeh, la Croix-Rouge, le Secours Populaire (Al-Isaaf El-Chaabi) et le Conseil Norvégien (signalé par la Municipalité mais qui est hors fonction actuellement). Parmi les autres centres, trois établissements sont reliés aux Ministères des Affaires Sociales et de l'Intérieur, un centre appartient à une agence internationale et deux sont fondés par des partis politiques. En plus, deux dispensaires ont été établis par des associations religieuses dont un est aussi lié au MAS (Ministère des Affaires Sociales). Pourtant, le seul dispensaire fondé par un médecin ouvre l'après-midi et n'a pas été mentionné par aucune des femmes enquêtées

Carte 3 - Répartition des dispensaires selon le type de direction

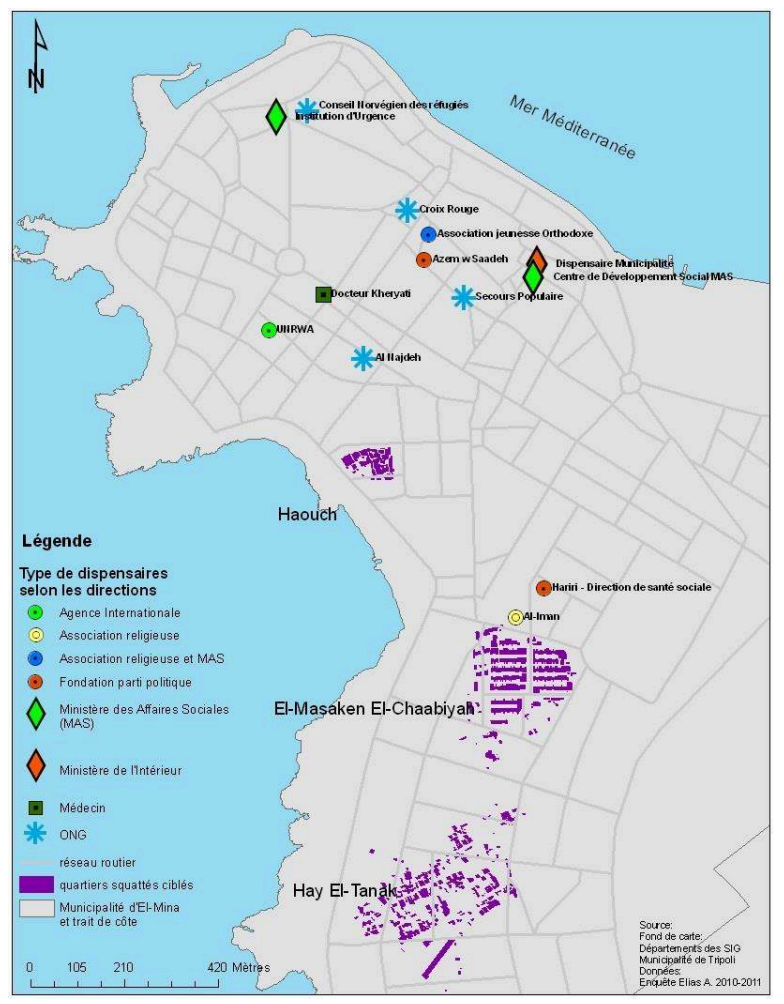

Source : Elias A.

Généralement, les dispensaires sont les structures les plus fréquentées par les habitants des quartiers squattés. Ces dispensaires, dont les activités consistent à offrir les soins primaires, ont su comment attirer les populations les plus défavorisées de la ville. Figurant parmi leurs priorités, les sessions de sensibilisation organisées sur place initient les mères aux comportements appropriés visant leur protection lors de la grossesse et la protection de leurs enfants au cours de leurs premières années de vie. La plupart des établissements de soin visités effectuent des conférences visant aussi l'hygiène personnelle des femmes, la nutrition des enfants et l'importance de la vaccination. La présence des infirmières dans les centres de soin est primordiale pour l'éducation des parturientes. Ainsi, les brochures et les « dépliants avec des schémas à emporter " sont nécessaires pour sensibiliser les femmes sur les bons comportements au cours des périodes pré et postnatale. Les mères qui n'ont pas un niveau d'étude élevé auront des ressources visuelles qui seront des guides de bonne conduite envers leur santé et celle de leur enfant à naître. Dans les dispensaires et les cliniques qui offrent 
des services aux femmes enceintes, «les conférences individuelles ou en groupe sont très bénéfiques (Schult, D., 1978) ». Les équipes médicales opérant dans ces structures effectuent aussi des campagnes de vaccination dans les écoles ainsi que dans les quartiers défavorisés de la ville.

Les infirmières rencontrées à la Croix Rouge, par exemple, ont déclaré que le dispensaire garde les coordonnées des individus qui s'y rendent et essayent de rappeler les mères à chaque fois que la prochaine consultation du gynécologue s'approche ou le prochain vaccin de l'enfant est dû. Dans ce centre, chaque enfant doit subir un checkup général chez le médecin, avant d'être vacciné. En plus des offres financières, ce dispensaire donne des vêtements et du lait aux femmes dans le besoin.

Les centres de soins se situent dans quatre zones tampons tout autour des quartiers squattés (cartes $4 \mathrm{a}, 4 \mathrm{~b}, 4 \mathrm{c}$ et $4 \mathrm{~d}$ ). Nous remarquons ainsi que le quartier le plus éloigné est Hay El-Tanak alors que Haouch est le plus proche des centres situés dans la ville. En effet, l'hôpital qui aurait été le plus proche ( Housseini, voire carte 6) a été fermé en 1995. Un autre hôpital qui se situe à cheval entre les villes de Tripoli et d'El-Mina est pratiquement le plus éloigné de tous les autres centres visités. Cette structure, qui a été fermée en 2009 (au cours de l'enquête ou quelques mois avant) pour des travaux de rénovation était un hôpital gouvernemental dédié aux services de maternité. Plusieurs gynécologues-obstétriciens y situèrent leurs cabinets libéraux. A l'origine, il a été fondé en 1970 par un gynécologue, le Dr. Ghossein, sous le nom de «l'hôpital de la mère et de l'enfant ». En 1975, date de déclenchement de la guerre civile libanaise, le fondateur qui résidait dans le même bâtiment le confia au gouvernement des Pays-Bas qui l'a dirigé sous le nom d'Orange Nassau ${ }^{17}$ jusqu'en 2003. A partir de cette date, l'hôpital fut réquisitionné pas le gouvernement Libanais sous la direction du Ministère de la Santé Publique.

79 En consultant les cartes $4 \mathrm{a}, 4 \mathrm{~b}, 4 \mathrm{c}$ et $4 \mathrm{~d}$, nous remarquons qu'un grand nombre des dispensaires les plus fréquentés par les habitants des quartiers ciblés se situent dans le centre de la ville. Pour accéder à ces structures, il faut presque 15 minutes de marche depuis Hay El-Tanak et une durée moindre pour les habitants de Haouch et du quartier d'El-Masaken. En revanche, la totalité des cabinets des gynécologues (et / ou obstétriciens) qui sont consultés pour le suivi de la grossesse sont localisés dans la ville de Tripoli. Mais ces spécialistes sont recrutés ou travaillent bénévolement dans les dispensaires de la ville d'El-Mina et dans l'hôpital d'Orange Nassau.

En fonction des localisations géographiques des centres de soin identifiés nous procéderons à la mesure de l'accessibilité aux services de soins nécessaires. Ceci aidera dans l'analyse des choix différenciés adoptés par les populations des quartiers squattés lors du recours aux soins. Cependant, les types d'offres effectuées par ces structures pourraient constituer des facteurs d'attraction ou de répulsion des populations de ces territoires défavorisés. 
Carte 4a - Les distances séparant les quartiers ciblés et les centres de soin fréquentés - les trois quartiers ciblés

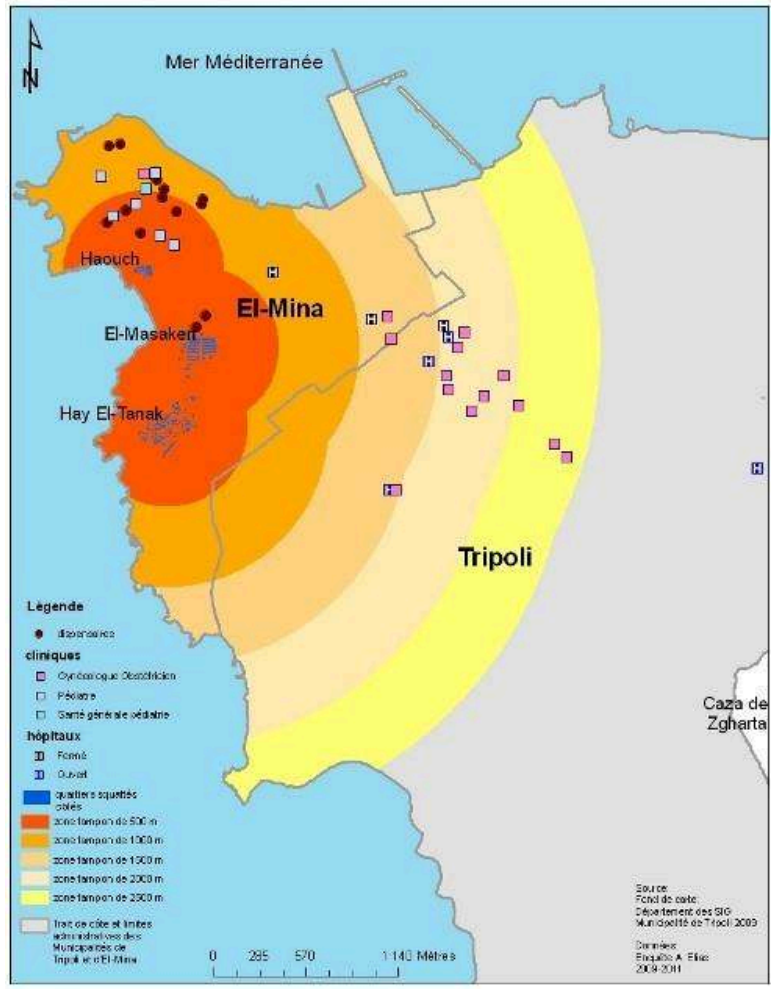

Carte $4 b$ - Les distances séparant les quartiers ciblés et les centres de soin fréquentés - Quartier de Hay El-Tanak

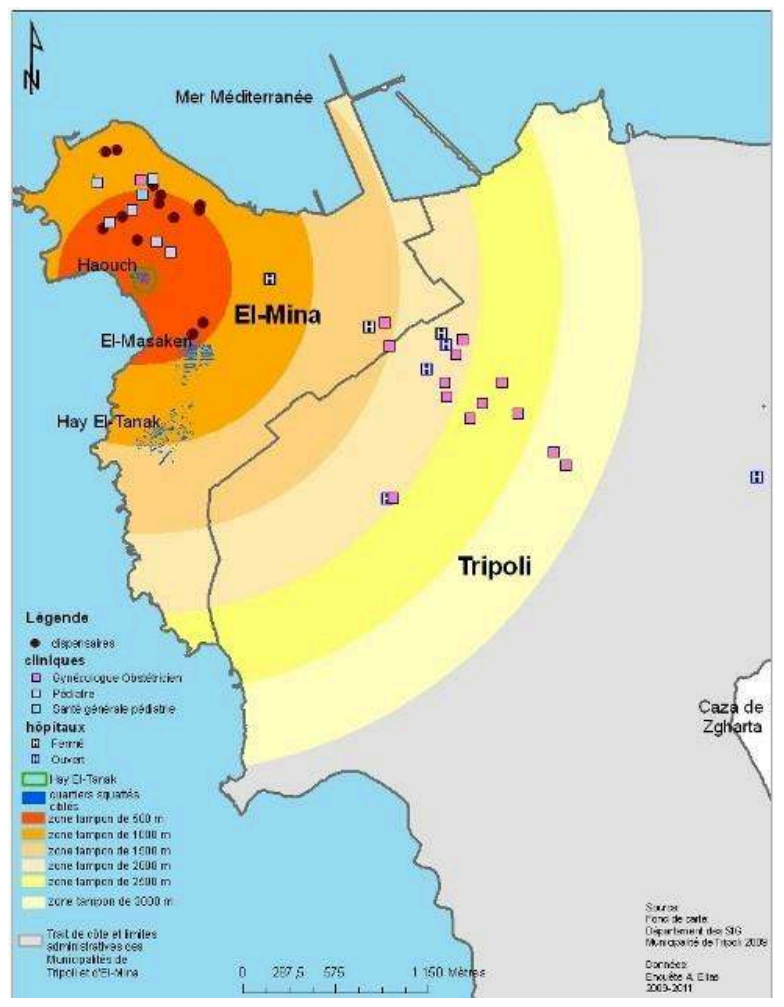


Carte 4c - Les distances séparant les quartiers ciblés et les centres de soin fréquentés - Quartier d' El Masaken El-Chaabiyah

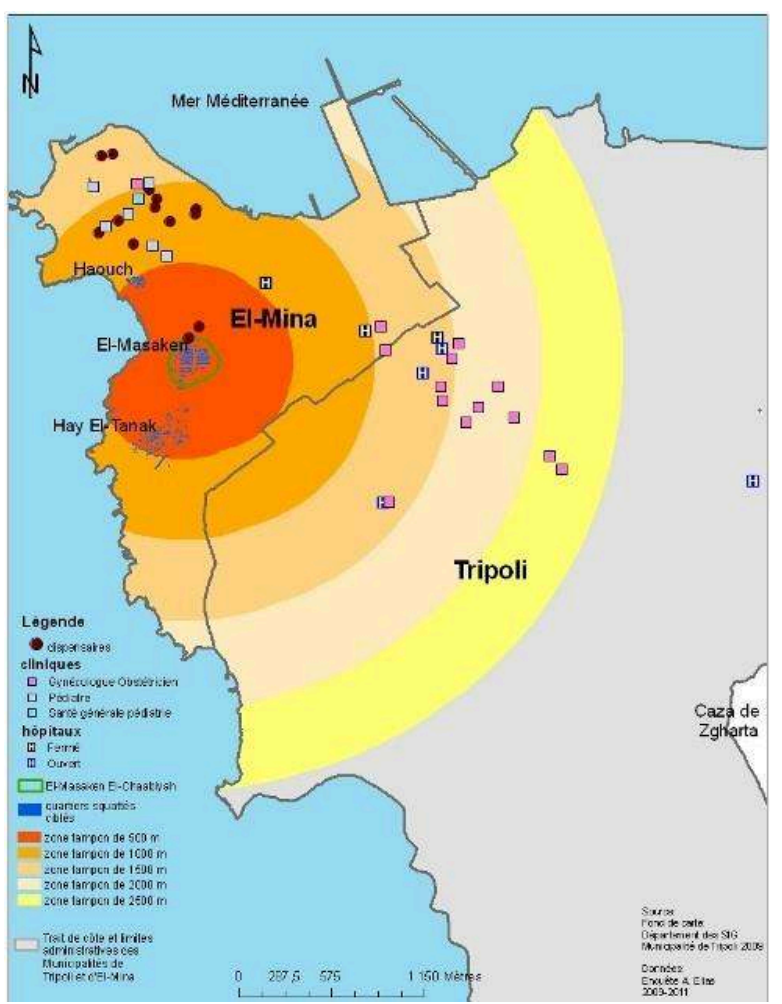

Carte $4 d$ - Les distances séparant les quartiers ciblés et les centres de soin fréquentés - Quartier de Haouch

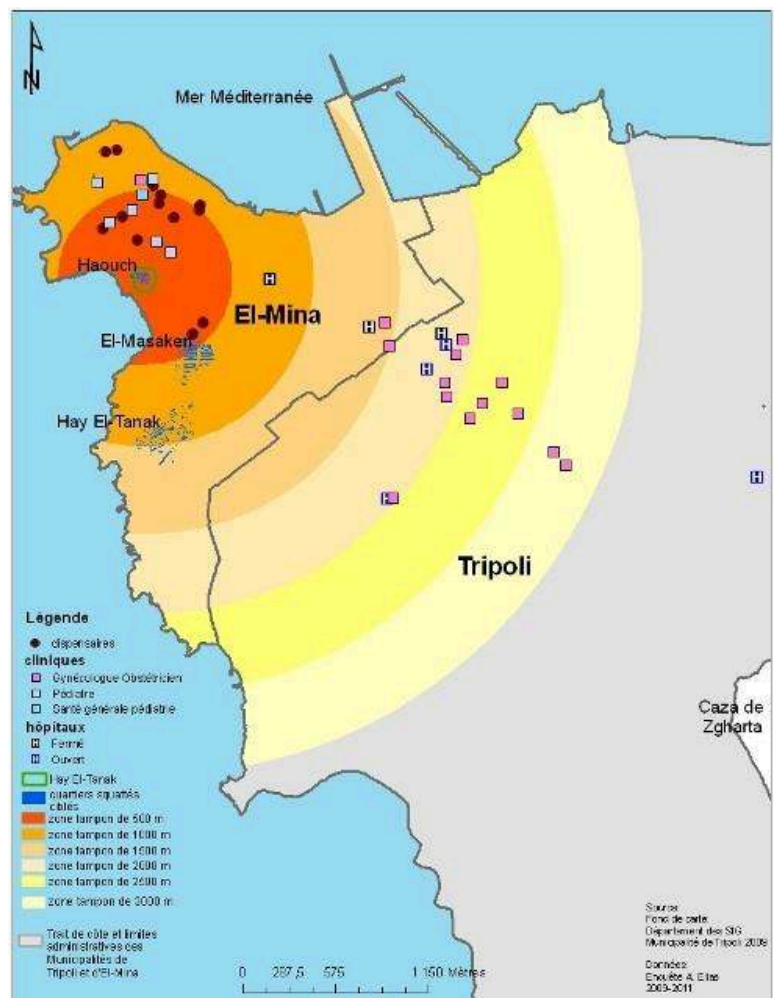




\section{Accessibilité differenciée aux soins relatifs à la mère et à l'enfant}

81 La proximité ne signifie pas accessibilité puisque d'autres paramètres, souvent d'ordre économique et social, entrent aussi en jeu. En effet, «l'accessibilité physique est la capacité de déplacement entre la localisation du patient et des points d'offre. L'accessibilité considère à la fois la mobilité des patients et les ressources en transport mobilisables, le trajet à parcourir en temps, en distance et en coût (Barlet M. et al, 2012)».

82 Pour calculer l'accessibilité aux centres de soin de la ville d'El-Mina nous nous sommes inspirés de l'indice d'accessibilité de Shimbel. Les trajets effectués vers les structures de soin se faisant à pieds, une carte du réseau de connexité entre quartiers et centres de santé a été développée, de laquelle dérive une matrice des écarts. L'écart dans ce cas est la distance en mètres du trajet le plus court effectué à pieds par les demandeurs de soin, sachant que la majorité des ménages enquêtés ne sont pas motorisés et que les quartiers ne sont pas bien desservis par les voitures services (voitures de transport en commun). Pour les cabinets libéraux situés à Tripoli, à l'extérieur des limites administratives d'El-Mina, les habitants des quartiers squattés ne peuvent pas s'y rendre à pieds. Comme présenté dans la méthodologie, les femmes enquêtées ont communiqué les noms des médecins ou des centres de soin fréquentés. Sur la carte 5 , nous avons localisé tous les centres de soin évoqués lors de l'enquête.

83 Selon la carte 5 de connexité il paraît que l'indice d'accessibilité le plus élevé est relatif au gynécologue S. M. (IA = 45,7) dont le cabinet privé est situé à la limite entre Tripoli et El-Mina. Concernant les pédiatres, le cabinet de Z. A. est le plus accessible également selon l'indice d'accessibilité de Shimbel. Ces structures sont les plus accessibles parmi tous les centres de soins inclus dans la matrice des écarts. Cependant, l'établissement, figurant comme le plus accessible en distance géographique, n'est pas le plus visité par les femmes pour suivre leurs grossesses.

84 Se référant à la carte 6, nous remarquons que les femmes habitant à Hay El-Tanak se dirigent surtout vers l'hôpital Orange-Nassau (six femmes), aussi dénommé l'hôpital de la mère et de l'enfant, pour le suivi de leur grossesse. Des fréquences moindres (quatre femmes) se rendent chez S. M. identifié comme le plus accessible. D'autres femmes de ce quartier préfèrent selon des fréquences variées des cabinets privés situés dans la ville de Tripoli. Au quartier de Haouch (carte 7), les femmes visitent principalement les cabinets des gynécologues se trouvant à Tripoli avec des fréquences égales. Cependant, la majorité des femmes du quartier d' El Masaken El-Chaabiyah se rendent aussi dans des cabinets libéraux situés à Tripoli. Comme le montre la carte 8 , dix femmes vont chez $\mathrm{M}$. H. qui avait aussi des permanences dans le centre de soin primaire Al-Iman situé dans le même quartier. Mais ces femmes fréquentent son cabinet à Tripoli, accessible uniquement par voiture, où plus de temps est consacré aux patientes. Ce fait est aussi confirmé par la spécialiste.

En comparant les résultats de l'IA obtenus aux choix effectués par les habitants des quartiers squattés, nous remarquons que la proximité géographique n'a pas un impact majeur sur les destinations choisies par les femmes enceintes pour suivre leur grossesse. Divers facteurs orientent ces choix qui sont liés à la qualité des soins, à la confiance envers le spécialiste et au montant de la consultation. 
En nous basant sur les déclarations des femmes rencontrées, celles-ci fréquentent les centres où les prix de consultation sont les moins chers. Mais aussi où les services offerts sont spécialisés et de bonne qualité. Dans ces centres, les tarifs de consultation varient entre 5,000 L.L. ${ }^{18}$ (2.5 euros) et 15,000 L.L. (7.5 euros). Selon leurs affirmations, les femmes choisissent le médecin qui a un bon accueil même s'il se situe plus loin. Celles-ci préfèrent aussi se rendre chez des gynécologues femmes et ce pour des raisons culturelles. Dans la majorité des cas ce sont les hommes qui imposent à leurs épouses ces choix qui sont le résultat de croyances religieuses conservatrices.

Carte 5 - Accessibilité aux centres de soins - (Elias A. 2009 - 2010)

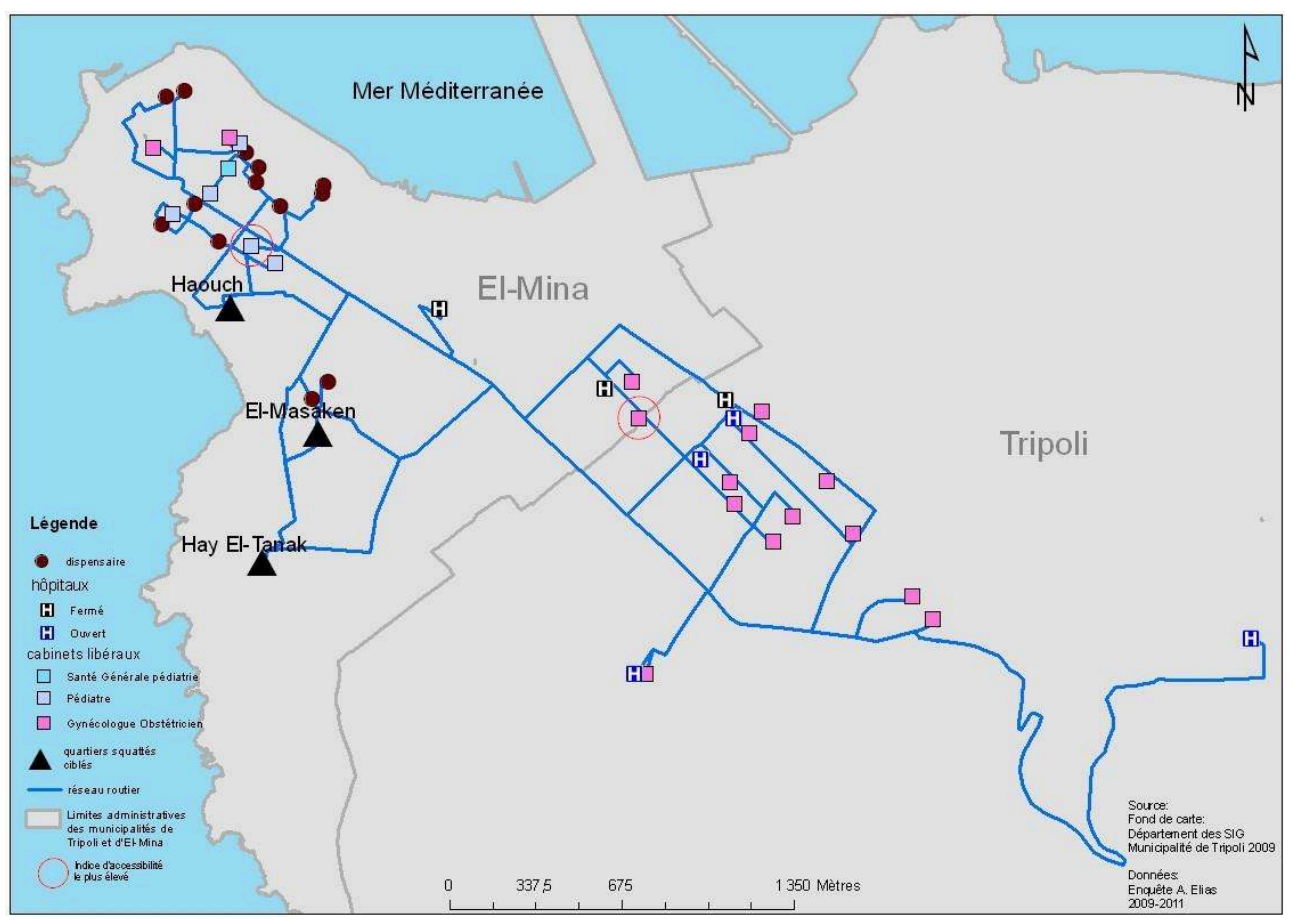


Carte 6 - Suivi de grossesse des femmes habitant le quartier de Hay El-Tanak (les centres de soin les plus fréquentés)

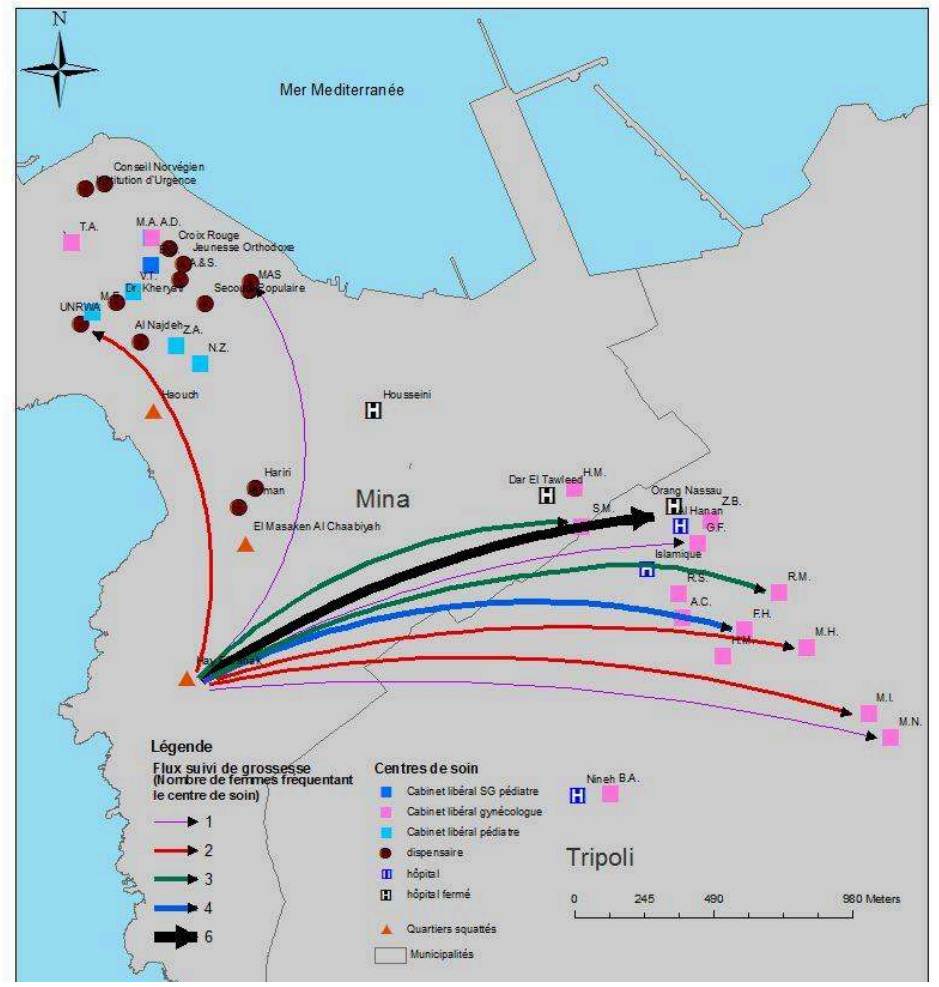

Carte 7 - Suivi de grossesse des femmes habitant le quartier Haouch (les centres de soin les plus fréquentés)

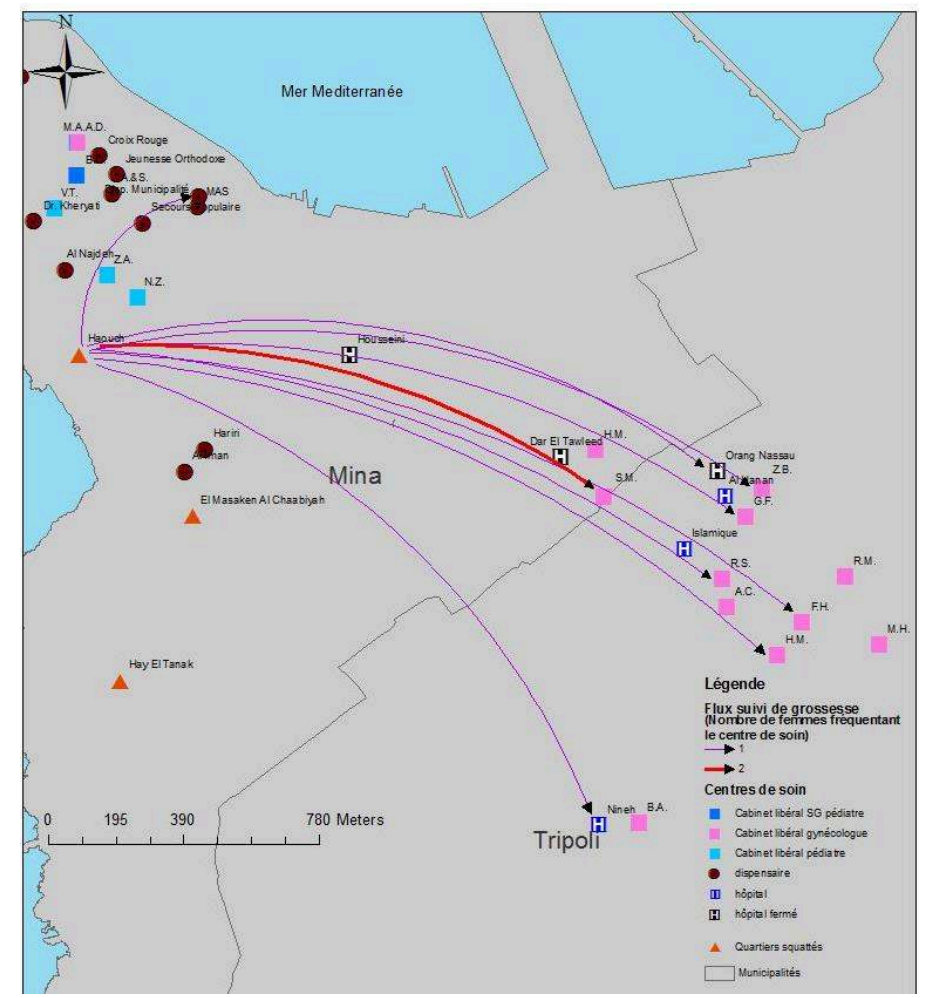


Carte 8 - Suivi de grossesse des femmes habitant le quartier d'El-Masaken (les centres de soin les plus fréquentés)

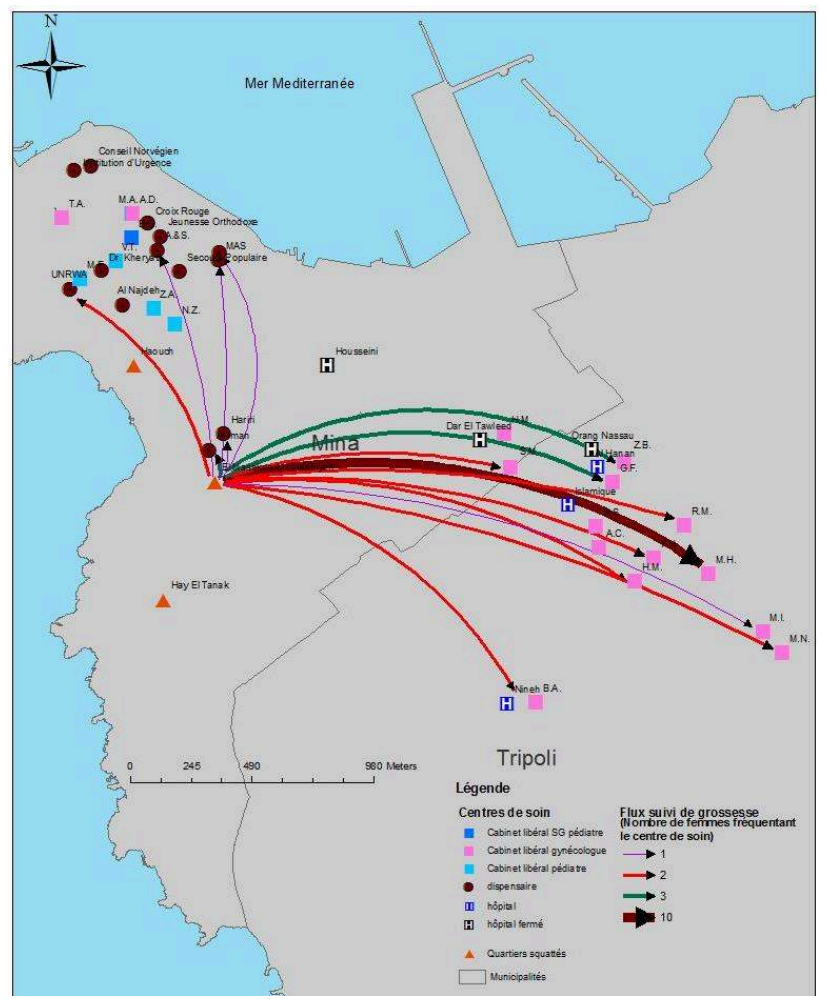

87 Quant à la santé des enfants, lorsque des symptômes de fièvre, de diarrhée ou de toux apparaissent, les mères adoptent d'autres choix de soins. Lors des trois types de symptômes qui ont été déclarés chez les enfants de moins de cinq ans, les mères habitant à Hay El-Tanak n'ont pas eu recours aux centres de soins : $32 \%$ lors des fièvres, $21 \%$ lors des diarrhées et $30 \%$ lors des toux (Tableau 3). Elles se sont contentées de l'automédication avec des remèdes déjà existant à la maison puisque certaines considéraient que le médecin n'est pas nécessaire dans ces cas.

Ces pourcentages sont moins élevés dans les deux autres quartiers, plus proches du centre de la ville. Ces symptômes dont les prévalences sont élevées, sont probablement récurrents vue les conditions de vie insalubres vécues, ce qui rend difficile de supporter le coût des consultations médicales. Néanmoins, dans les trois quartiers les parents qui ont recherché le soin pour remédier à la fièvre de leurs enfants, se sont dirigés vers un médecin comme premier choix. Lorsqu'il s'agissait d'une diarrhée, ces parents se rendaient soit au dispensaire de Tebbaneh (quartier situé dans la ville de Tripoli), soit au centre de soin primaire d' Al-Iman, dans le quartier d' El Masaken El-Chaabiyah . En fait, la destination vers les pharmacies occupe une importance similaire à celle des dispensaires. Certains parents se sont rendus aussi vers des hôpitaux, ceci est un indicateur de cas sévère comme il peut être une conséquence de l'indisponibilité des dispensaires et des cliniques pendant les soirées. 
Tableau 3 - Les choix de soins effectués lors des symptômes de Fièvre, de Diarrhée et de Toux

\begin{tabular}{|c|c|c|c|c|c|c|c|}
\hline \multicolumn{2}{|l|}{$N$ Total $=139$} & \multicolumn{2}{|c|}{ Hay El-Tanak } & \multicolumn{2}{|c|}{ Haouch } & \multicolumn{2}{|c|}{$\begin{array}{l}\text { El-Masaken } \\
\text { El-Chaabiyah }\end{array}$} \\
\hline Symptômes & Destination & $\begin{array}{l}N= \\
47\end{array}$ & $\%$ & $\begin{array}{l}N= \\
23\end{array}$ & $\%$ & $\begin{array}{l}\mathrm{N}= \\
69\end{array}$ & $\%$ \\
\hline \multirow{5}{*}{$\begin{array}{c}\text { Fièvre } \\
\text { Qu'avez-vous fait? }\end{array}$} & Automédication & 15 & 32 & 2 & 9 & 8 & 12 \\
\hline & Dispensaire & 1 & 2 & 1 & 4 & 3 & 4 \\
\hline & Hôpital & 0 & 0 & 1 & 4 & 0 & 0 \\
\hline & Médecin & 9 & 19 & 2 & 9 & 10 & 14 \\
\hline & Pharmacie & 0 & 0 & 1 & 4 & 0 & 0 \\
\hline \multirow{6}{*}{$\begin{array}{l}\text { Diarrhée } \\
\text { Qu'avez-vous fait? }\end{array}$} & Rien & 1 & 2 & 0 & 0 & 0 & 0 \\
\hline & Automédication & 10 & 21 & 2 & 9 & 8 & 12 \\
\hline & Médecin & 4 & 9 & 1 & 4 & 3 & 4 \\
\hline & Pharmacie & 0 & 0 & 1 & 4 & 0 & 0 \\
\hline & Dispensaire & 0 & 0 & 0 & 0 & 2 & 3 \\
\hline & Hôpital & 2 & 4 & 0 & 0 & 2 & 3 \\
\hline \multirow{6}{*}{$\begin{array}{l}\text { Toux } \\
\text { Qu'avez-vous fait? }\end{array}$} & Rien & 2 & 4 & 1 & 4 & 2 & 3 \\
\hline & Automédication & 14 & 30 & 4 & 17 & 15 & 22 \\
\hline & Médecin & 4 & 9 & 2 & 9 & 3 & 4 \\
\hline & Pharmacie & 1 & 2 & 0 & 0 & 1 & 1 \\
\hline & Dispensaire & 0 & 0 & 1 & 4 & 1 & 1 \\
\hline & Hôpital & 0 & 0 & 1 & 4 & 0 & 0 \\
\hline
\end{tabular}

Pour assurer la vaccination de leurs enfants (Carte 9) les mères de Hay El-Tanak optent pour la Croix-Rouge (12 enfants) bien qu'il soit le plus éloigné du quartier. Toutefois, il est important de mentionner que les quartiers squattés ciblés sont parmi les territoires couverts par la Croix-Rouge lors des campagnes nationales de vaccination. Comme le montre la carte 9, dans le quartier Haouch les enfants accompagnés par leurs parents (quatre enfants) se rendent au dispensaire d'Al-Najdeh à proximité du quartier (5 minutes de marche), puis à la Croix-Rouge (trois cas). Les parents du quartier d' $\mathrm{El}$ Masaken choisissent des lieux de soins différents. Le plus grand nombre (neuf enfants) fréquentent le dispensaire de la Fondation Hariri qui est le plus proche (5 minutes de marche). Pour justifier leurs choix, la plupart des mères ont déclaré choisir le lieu de vaccination le moins cher et où des aides financières sont effectuées. Parmi celles-ci il y a des mères qui se rendent à des centres où la vaccination est gratuite. Toutefois, un pourcentage non négligeable des enfants de ces quartiers n'ont pas pris les vaccins nécessaires à leur âge (43\%). 
Carte 9 - Destinations des mères pour vacciner leurs enfants âgés moins de 5 ans

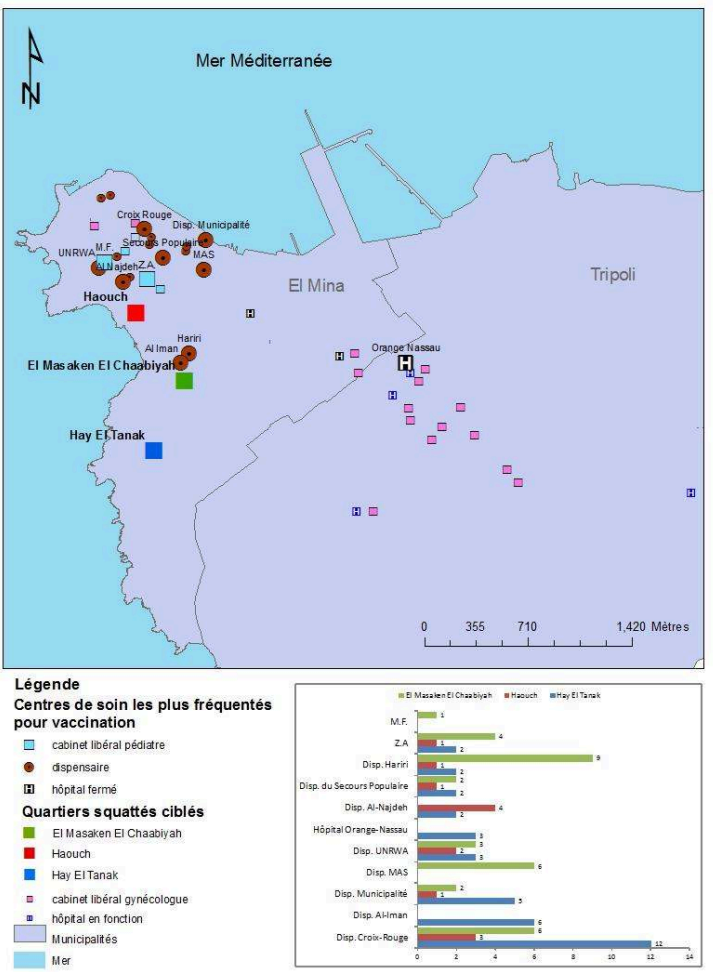

Source : Elias A. 2009 - 2010

90 Ainsi, plusieurs facteurs influent sur l'accessibilité aux soins des habitants des territoires squattés. La proximité géographique ne figure pas parmi les facteurs primordiaux stimulant l'accès au cours de la phase périnatale. D'autres variables d'ordre économique, social et qualité d'offre jouent aussi un rôle déterminant. Or, des contraintes sont toujours perçues dans l'accès aux soins nécessaires aux enfants après la phase périnatale. Ceci est lié à la négligence du recours au soin spécialisé due aux capacités financières réduites des ménages habitant dans les quartiers squattés et probablement au faible niveau d'instruction des mères.

\section{Mobilité influencée par les offres multiples et la prise en charge des soins}

Les entretiens menés avec les fournisseurs de soin ont révélé l'existence de prestations sociales visant à encourager les populations démunies d'accéder aux soins. Cette accessibilité facilitée est le résultat d'un contexte de solidarité qui règne dans la ville d'El-Mina qui engage plusieurs acteurs urbains dans le secteur de la santé. Ces derniers visent une minimisation des inégalités d'accès aux soins et souhaitent combler l'absence d'une couverture sanitaire universelle non assurée par l'Etat.

Ces acteurs sont non gouvernementaux, comme les organisations locales ou internationales, les médecins et les associations caritatives souvent religieuses et politiques. Ils sont même gouvernementaux comme la Municipalité et les Ministères qui ont financé la fondation de plusieurs dispensaires dans la ville. 
93 Ce réseau d'aide remplace partiellement la sécurité sociale et l'assurance de santé privée desquels les populations pauvres sont généralement exclues. Les types d'assistance effectués par les acteurs de ce réseau d'aide diffèrent d'une structure de soin à l'autre et se basent sur le niveau de besoin du demandeur de soin. Parmi les dispensaires visités, 2 centres ont déclaré offrir des soins gratuits à tous les patients, 3 autres ont révélé qu'ils assurent une couverture complète des familles démunies (tableau 4) et des personnes ayant un handicap physique. Le reste des dispensaires (tableau 4) offrent des médicaments gratuits, font des réductions de $50 \%$ aux frais de consultation, assurent une couverture complète des orphelins ou offrent des soins gratuits aux pêcheurs et aux réfugiés en particulier.

Tableau 4 - Types d'assistance effectuée par les dispensaires

\begin{tabular}{|l|l|}
\hline Types d'assistance & Fréquence \\
\hline Soins gratuits & 2 \\
\hline Couverture complète des personnes handicapées et familles démunies & 3 \\
\hline Offre de médicaments gratuits & 1 \\
\hline Réduction $50 \%$ & 1 \\
\hline Couverture complète des orphelins & 1 \\
\hline Soins gratuits aux pêcheurs et aux réfugiés & 1 \\
\hline
\end{tabular}

Source : Elias A. $2010-2012$

94 A leur tour, les médecins jouent le rôle de facilitateurs d'accès aux soins en fournissant parfois des services gratuits et en offrant les médicaments nécessaires à titre personnel. Deux des pédiatres les plus consultés par la population des quartiers squattés sont actuellement membres du conseil municipal de la ville d'El-Mina alors qu'une troisième faisait partie du comité municipal de santé et des affaires sociales pour plusieurs années. Ceci renforce leurs intentions de soutenir les populations des quartiers squattés et de minimiser la morbidité dans les territoires squattés. Or, concernant les vaccins qui ne sont pas couverts par le programme national de vaccination, ceux-ci ne sont pas offerts gratuitement par ces spécialistes.

95 Egalement, les associations religieuses (carte 10) ont créé un mécanisme d'aide aux ménages défavorisés dans la procuration des médicaments nécessaires. Celles qui sont les plus actives en matière d'assistance sanitaire de la ville sont Makarem El-Akhlaa' et le Conseil de la Paroisse de l'Eglise Saint-Georges. L'importance de ces aides est différente selon le quartier de résidence, Hay El-Tanak étant le territoire qui reçoit le plus d'assistance de ce genre.

96 Deux partis politiques tripolitains (carte 10) ont aussi installé un système d'assistance dans la ville, ceux-ci sont Azem et Saadeh et Safadi. Le premier a fondé un dispensaire dans la ville en 2011. Situé au centre, il couvre les factures des médicaments et une partie des frais de soins hospitaliers tels que les opérations chirurgicales. Les bénéficiaires sont sélectionnés après étude de leurs conditions socio-économiques. Le second est actif à partir d'une fondation caritative dont les aides principales se concentrent sur le financement de la facture des soins hospitaliers notamment les 
opérations chirurgicales des problèmes cardiaques, la cataracte et les césariennes, mais dans une petite proportion.

Carte 10 - Les associations localisées dans la ville d'El-Mina

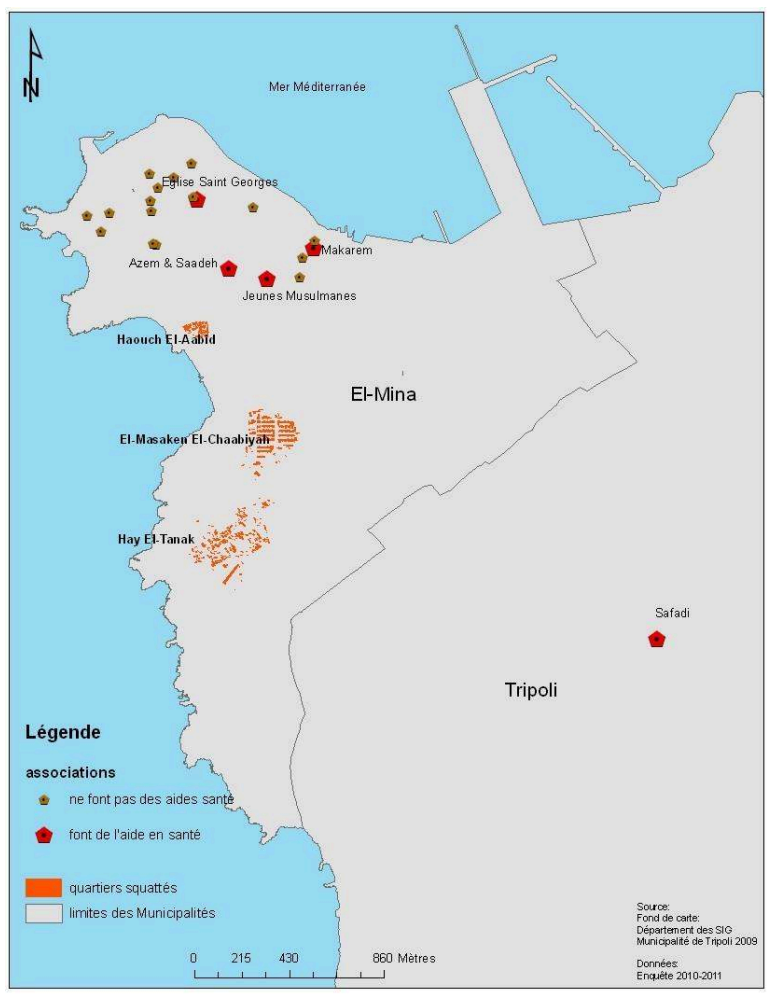

Source : Elias A. 2012 


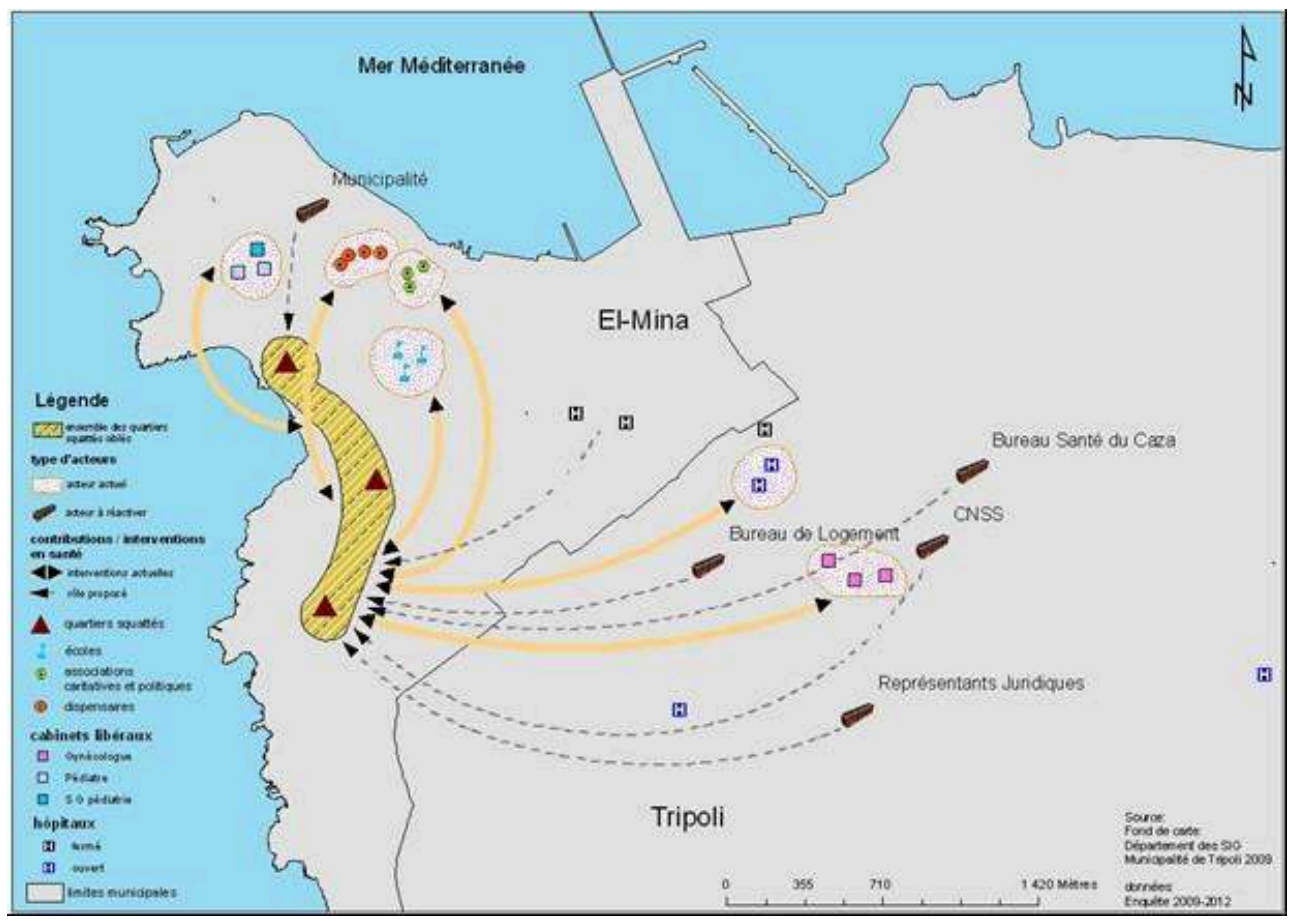

Source : Elias A.

Une carte récapitulative (carte 11) mettant en relief les actions qui ciblent la santé et les quartiers ayant des difficultés socio-économiques, y compris les quartiers squattés, a été élaborée. Celle-ci sert comme un modèle synthétique des données collectées pour essayer de comprendre les dynamiques spatiales et les facteurs influant sur la santé des habitants ciblés. D'une part, les dispensaires, les cabinets médicaux et les hôpitaux offrent des soins aux habitants des quartiers squattés tout en facilitant leur accès spatial et financier. D'autre part, les écoles primaires de la ville prennent des mesures protectrices exigeant le respect du calendrier national de vaccination pour les enfants qui y sont inscrits. A leur tour, les associations religieuses et politiques participent dans les facilitations financières des plus démunis aux soins. La Municipalité, comme représentant local du gouvernement, contribue à travers son dispensaire gratuit et ses programmes d'hospitalisation à la constitution de l'état de santé actuel des habitants des quartiers squattés.

Aussi, le syndicat des pêcheurs du Liban Nord, auquel sont affiliés les pêcheurs résidant dans la ville d'El-Mina, exerce un rôle dans les prestations sanitaires dans la ville d'El-Mina. Ce syndicat fondé en 1970 regroupe presque 50 pêcheurs habitant dans les quartiers squattés selon les déclarations de son président. En tant que membre, chaque pêcheur paie 24,000 L.L. (14 euros) régulièrement par an. En 2010, un protocole a été signé avec le dispensaire d' Al-Najdah Al-Chaabiyah permettant aux pêcheurs et à leurs familles d'avoir un accès gratuit aux soins dans cette structure. Si nécessaire, les patients sont transférés chez des spécialistes à l'extérieur du centre où ils sont soignés gratuitement. Avant janvier 2012, l'Association Azem \& Saadeh remboursait une partie des frais d'hospitalisation, comme le coût d'accouchement équivalent à 150,000 L.L. (88 euros), cette somme qui reste après la part couverte par le MSP. Mais les 
médicaments ne sont pas disponibles chez Al-Najdah Al-Chaabiyah puisque la caisse du Syndicat ne suffit pas.

99 Les activités des acteurs urbains facilitant surtout l'accès financier au soin s'inscrivent dans un contexte social libanais qui est caractérisé par une solidarité avec les plus nécessiteux. Dans son ouvrage sur le "service de la santé au Liban » le père DUCRUET affirme que les dispensaires déjà nombreux dans les années 40 étaient des «œuvres caritatives financées par les Wakfs, fondations des communautés religieuses chrétiennes et musulmanes (Ducruet, 2009)».

Dans les années 50 "deux institutions ont joué un rôle déterminant dans l'évolution des centres de santé " l'Office de Développement Social (O.D.S.) créé en 1959 et le Mouvement Social fondé en 1957 (reconnu par le Ministère de l'Intérieur en 1963). Celles-ci ont participé à la transformation du dispensaire d'un centre de bienfaisance à un «centre médico-social» ayant un rôle précis dans un système de santé. L'O.D.S. " était chargé d'établir un programme pour le développement social du Liban et d'en contrôler la réalisation, de proposer et exécuter les projets sociaux pris en charge par l'Etat lui-même, de participer à l'aide de subventions et de prêts à la réalisation et au renforcement de projets sociaux privés ». À son tour, le Mouvement Social a été créé avec la «prise de conscience» des conditions de vie défavorisées régnant dans certaines régions du Liban. Ces deux organisations dont les activités étaient sociales et médicales étaient précédées par l'Ordre Souverain de Malte qui «a ouvert son premier centre médico-social en 1956 » et par «La Croix-Rouge Libanaise reconnue d'utilité publique en $1946 \%$.

101 La guerre libanaise qui a commencé en 1975 et qui a duré 15 ans a provoqué des vagues de déplacements internes. Ceci a participé au développement au cours et après la guerre du rôle de ces organisations visant l'aide humanitaire au Liban pour combler l'absence du rôle de l'Etat dans plusieurs secteurs vitaux, dont la santé.

\section{Conclusion}

102 L'étude effectuée à l'échelle locale des quartiers squattés de la ville d'El-Mina a révélé qu'un pourcentage élevé des femmes qui résident dans ces territoires font suivre régulièrement leur grossesse à partir du premier mois malgré la situation socioéconomique défavorisée, particulièrement dans le quartier d'El-Masaken El-Chaabiyah qui prend la forme d'un lotissement plus organisé et qui est doté de conditions de vie relativement meilleures. Dans les trois quartiers squattés, qui sont apparus à des dates distinctes et dans des endroits séparés de la ville d'El-Mina, la grande majorité des femmes qui suivaient leurs grossesses n'ont pas eu des naissances prématurées moyennes et n'ont pas déclaré avoir eu des décès néonataux. Ces comportements " prophylactiques » aboutissent à la protection de la femme et de l'enfant au cours de la phase périnatale bien que les moyens financiers et les ressources économiques dont disposent ces populations sont très minimes.

103 Le calcul de l'indice d'accessibilité a montré que la proximité géographique des structures de soin ne joue pas un rôle majeur dans les choix des destinations de soins effectués par les femmes enceintes. Plusieurs facteurs orientent ces choix et sont liés à la qualité des services offerts, à la confiance envers les spécialistes et au montant de la consultation. Les facteurs cités sont assurés par plusieurs acteurs urbains qui facilitent l'accessibilité aux soins des populations des quartiers squattés. Ces acteurs ont abouti à 
la formation d'un réseau d'aide qui rassemble des organisations locales et internationales, des médecins spécialistes, et des associations caritatives et politiques qui essayent de combler le manque des prestations supposées être assurées par le système de soin classique. Ce réseau socio-spatial intègre les populations pauvres dans le système de soin en s'appuyant sur une offre multiple existant dans la ville d'El-Mina qui prouve être plus fonctionnelle ainsi.

104 Cependant, après la phase périnatale plusieurs contraintes limitent l'accès au soin des enfants. Puisque, après cette phase, la santé des enfants est de moins en moins suivie par un soin spécialisé. Les mères ont recours à l'automédication notamment à Hay ElTanak le plus démuni et le plus éloigné parmi les trois quartiers ciblés. Ceci est associé à la négligence du soin due d'abord aux difficultés financières et probablement au faible niveau d'étude des femmes habitant dans les quartiers squattés. D'où l'importance des activités de sensibilisation des mères et des suivis plus accentués qui s'avèrent essentiels de la part des personnels des centres de soins de la ville.

Bien que tous squattés, chacun des territoires ciblés par cette étude possède une histoire différente qui a abouti à un accroissement, une morphologie et des conditions de vie différentes. Ceci s'est traduit par des états de santé distincts dans certains cas et des comportements parfois différenciés dans le recours aux soins nécessaires.

En même temps L'observation de certaines caractéristiques du réseau d'aide en santé et les facilitations fournies aux populations pauvres de la ville, non spécifiquement celles des quartiers squattés, a permis de lire une approche de solidarité qui a commencé à se développer au Liban avec l'apparition des premiers habitats fragiles et qui remplace partiellement le rôle de l'Etat dans la sécurité sociale et sanitaire. Or, ces interventions de type politique, étatique, municipal ou religieux sont isolées, personnelles, indépendantes et certaines demeurent inaccessibles aux personnes qui ne veulent pas solliciter l'aide. Celles-ci englobent parfois des intérêts politiques et religieux ce qui pourrait créer d'autres types d'inégalité parmi les populations nécessiteuses ellesmêmes. Ces interventions ont lieu en aval, après que le problème de santé surgisse. Par conséquent elles n'éliminent pas l'existence des aléas qui persistent et qui affectent le bien-être des habitants particulièrement les enfants qui souffrent des symptômes de diarrhée, de fièvre et de toux dont les proportions sont élevées. Ainsi, l'amélioration des conditions de vie, particulièrement de l'habitat et des infrastructures sanitaires est primordiale.

107 De même, ces comportements protecteurs requièrent parfois des dépenses supplémentaires de déplacement. Par conséquent, se basant sur les critères d'attraction chez les populations étudiées, une synergie entre la couverture financière des plus démunis, la proximité des structures de soin nécessaires, la facilitation du déplacement et la qualité de soin est recommandée. Ainsi, les nouveaux établissements de santé devraient favoriser les périphéries, plus particulièrement les environs de Hay El-Tanak qui est l'un des quartiers les plus excentrés de la ville.

108 Aussi, faut-il rappeler que notre étude effectuée entre 2009 et 2012 dévoile la situation des quartiers squattés avant l'installation des réfugiés syriens dans ces parties de la ville. Une étude plus profonde sur le changement des conditions de vie et de l'impact des vulnérabilités jaillissantes dans ces territoires sur la santé des résidents, libanais et réfugiés syriens, s'avère aujourd'hui essentielle. Ceci dans le but de focaliser les efforts sur la rectification des politiques d'intervention notamment dans les camps informels de réfugiés. 


\section{BIBLIOGRAPHIE}

Agier M., 1999, L'invention de la ville, banlieues, townships, invasions et favelas, Paris : Edition des Archives Contemporaines.

Arab League, Ministry of Social Affairs (Lebanon), Central Administration of Statistics, 2004, Lebanon Family Health Survey (PAPFAM). 380 pages.

Arslanian J., 1961, Logement et santé. Article dans le cadre des Semaines sociales du Liban, l'Habitat dans La vie libanaise, 17-27 avril 1961, éditions les lettres orientales Beyrouth 1961, p. 25-47.

Baghdadi S. (dir) 2005, « étude de la réalité socio-économique des villes de Tripoli, El-Mina et AlBaddawi », 1ère phase, Bureau Technique des Villes et Gouvernements Locaux, programme de développement local, Union des Municipalité de Fayhaa. P. 3. (traduit de la langue l'arabe)

Barlet M., Coldefy M., Collin C. et Lucas Gabrielli V., 2012, Accessibilité Potentielle Localisée (APL) : une nouvelle mesure de l'accessibilité aux soins appliquée aux médecins généralistes libéraux en France. IRDES, Document de Travail nº 51, 56 pages.

Bicqrd, D., Jusot, F., Tubeuf, S., 2010, Les modes de vie: un canal de transmission des inégalités de santé ?, Questions d'économie de la santé IRDES ; n 154 - Mai 2010 ; 6 pages ; http://www.irdes.fr/ Publications/2010/Qes154.pdf

Charreire H., Combier E., 2006, Équité socio-spatiale d'accès aux soins périnatals en milieu urbain: utilisation des techniques géographiques pour la planification sanitaire. Espace populations sociétés 2006 / 2-3 Espace urbain et santé. P 313-327. https://eps.revues.org/1699?lang=en paragraphe 51

Chen B., Cammett M., 2012, Informal politics and inequity of access to healthcare in Lebanon. International Journal for Equity in Health. (c) 2012 Chen and Cammett ; licensee BioMed Central Ltd. 8 pages.

Cities Alliance and Al-Fayhaa, 2011, Sustainable Development Strategy, Final Consolidation Report ; version .1, Al-Fayhaa Union of Municipalities, 236 pages.

Colin C., 1991, La santé des femmes défavorisées : un quotidien difficile, un avenir compromis femmes et santé, aspects psychosociaux, sous la direction de Queniart A., Boucherville : éditeur Gaëtan Morin.

Denantes M., Chevillard M., Renard J.F., Flores P., 2009, Accès aux soins et inégalités sociales de santé en médecine générale. Exercer La revue française de médecine générale. Volume $20 / \mathrm{n}^{\circ} 85$, p. 22-26.

Devlieger, H., Martens, G. \& Bekaert, A., 2005, Social Inequalities in Perinatal and Infant Mortality in the Northern Region of Belgium (the Flanders). Eur J Public Health 15, 15-9

Ducruet, J., s.j. 2009, Le service de la santé au Liban, histoire, législation, institutions, état de santé de la population et prestations de soins, système et politique de la santé; Presses de l'Université Saint-Joseph ; 327 pages

H. Peters D., Garg A., Bloom G., Walker D., Brieger W., and Rahman H., 2008, Poverty and Access to Health Care in Developing countries. Ann. N.Y. Acad. Sci. 1136: 161-171 New York Academy of Sciences, p. 161-171. Doi: 10.1196/annals.1425.011

Habib, R.R., Mahfoud, Z., Fawaz, M., Basma, S.H., Yeretzian, J.S., 2009, Housing quality and ill health in a disadvantaged urban community ; Elsevier Public Health 123, pages 174-181. 
Le Goffe, E., Sechet, R., Davido, O., 2010, « Penser la santé dans la gestion et l'aménagement des espaces urbains : un projet de recherche sur les Villes-Santé en Bretagne »; ESO - RENNES ; ${ }^{\circ}$ 30, décembre 2010 ; p. 33-41

L'Institut de gestion de la santé et de la protection sociale, l'Université Saint-Joseph, 2012, Recueil national des statistiques sanitaires au Liban, en collaboration avec le Ministère de la Santé Publique et l'Organisation Mondiale de la Santé, Beyrouth. http://www.igsps.usj.edu.lb/docs/ recherche/recueil12fr.pdf

Ménard B., 2002, Questions de géographie de la santé. in l'Espace Géographique 3/2002 (tome 31), p. 264-275, (en ligne) http://www.cairn.info/revue-espace-geographique-2002-3-page-264.htm

Merlin, P. et F. Choay (dir), 1996, Dictionnaire de l'urbanisme et de l'aménagement, Paris : Presses universitaires de France, deuxième édition revue et augmentée ( $1^{\text {ère }}$ édition 1988).

Ministère de la Santé Publique, 2004, Recueil national des statistiques sanitaires au Liban, en collaboration avec la Coopération Italienne pour le Développement et l'Université Saint-Joseph, Beyrouth.

Ministère de la Santé Publique, 2012, Recueil national des statistiques sanitaires au Liban, en collaboration avec la Coopération Italienne pour le Développement et l'Université Saint-Joseph, Beyrouth.

Nisar N., White F., 2003, Factor affecting the utilization of Antenatal care among reproductive age women (15-49 years) in an urban squatter settlement of Karachi. JPMA (Journal of Pakistan Medical Association) Vol 53/nº 2.

OMS, 2009, Les femmes et la santé, la précarité d'aujourd'hui le programme de demain, éditions de l'OMS.

OMS, 2000, Barton, H, Tsourou, C. « Urbanisme et santé un guide de l'OMS pour un urbanisme centré sur les habitants "; la version française a été publiée en 2004 par S2D Association internationale pour la promotion de la santé et du Développement Durable ; 193 pages.

OMS, 2010, Rapport sur la santé dans le monde, le financement des systèmes de santé, le chemin vers une couverture universelle, sous la direction de Etienne, C. et Asamoa-Baah, A., éditions de l'OMS, Genève, Suisse, 120 pages.

Picheral, H., 2001, Dictionnaire raisonné de géographie de la santé ; Université Montpellier III-Paul Valéry, 308 pages.

Pumain D., Saint-Julien T., 1997, L'analyse spatiale, localisations dans l'espace. Collection cursus. Armand colin. 167 pages. P. 96.

Salti, N., Chabban, J., Raad, F., 2010, Health Equity in Lebanon : a microeconomic analysis; International Journal for Equity in Health 2010, 9:11; 21 pages

Shah, A., et al., 2007, Methodological considerations in implementing the WHO global survey for monitoring maternal and perinatal health; publication: Bulletin of the World Health Organization; type: Research Article ID: 06-039842; Article DOI: 10.2471/BLT.06.039842

Schult, D., 1978, « L'Infirmière et la périnatalité » traduit par L. Dumas-Rivest ; les éditions HRW ; Bibliothèque nationale du Québec. Page 104

Semaines sociales du Liban « l'Habitat dans la vie libanaise »; 17-27 avril 1961 ; éditions les Lettres Orientales Beyrouth $1961 ; 137$ pages, p. 36

Veyret Y., 2003, Les risques, dossiers des images économiques du monde, collection dirigée par Gamblin A., Paris : SEDES. 
Vallée, J., Fournet F., Meyer P-E, Harang M., Pirotand F., Salem G., 2006, « Stratification de la ville de Ouagadougou (Burkina Faso) à partir d'une image panchromatique Spot 5 : Une première étape à la mise en place d'une enquête de santé ", Espace populations sociétés, 2006/2-3, p 393-401.

\section{NOTES}

1. Fayhaa signifie la ville odorante : «En raison du parfum autrefois dégagé d'agrumes en fleurs, plantations qui recouvraient l'ensemble de la plaine littorale et qui assurèrent durant plusieurs siècles une partie de la prospérité économique de la cité. » http://dictionnaire.sensagent.com/tripoli+(liban)/fr-fr/ - cite_note-0

2. Enquête effectuée dans les quartiers squattés d'El-Mina ; 2009-2010

3. «L'activité foncière et immobilière au Liban, dénuée de toute régulation sérieuse, représente un cas extrême d'exclusion sociale par la spéculation haussière... un cas extrême d'insécurité des personnes et des biens dans des constructions réalisées dans des conditions de danger sanitaire et physique "; (Awada, 2006)

4. Surface calculée sur ArcGIS10.

5. Source : Dénombrement effectué au cours de l'enquête (hiver - printemps) 2009: A. Elias; un nombre qui n'est pas fixe et qui change en permanence.

6. Terme arabe signifiant vergers.

7. Source : enquête hiver - printemps 2009-2010 Elias A.

8. Source : enquête hiver - printemps 2009-2010 Elias A.

9. Avant 37 semaines d'aménorrhée

10. Les nouveau-nés morts au cours de leurs 28 premiers jours de vie. La mortalité est considérée comme précoce si elle a lieu au cours des 7 premiers jours de vie

11. Ces campagnes de vaccination ont été répétées en 2013 - 2014 suite à des cas de polio qui ont surgi parmi les enfants réfugiés Syriens au Liban.

12. « Prospectus sur les établissements de santé au Liban (hôpitaux, centres de santé, et dispensaires) nombre, distribution et services "; République Libanaise - Ministère de la Santé Publique 2009 avec l'aide de l'UNICEF, 36 pages ; p. 5

13. idem.

14. Ib Idem, p. 229

15. Chiffres datant de 2012

16. Clinique est un terme utilisé au Liban pour désigner un cabinet libéral, il ne signifie pas dans notre cas une polyclinique mais un cabinet privé au médecin généraliste ou spécialiste.

17. Orange-Nassau : famille régnante des Pays-Bas de 1814 à 1948.

18. L.L. (Livre Libanaise) 


\section{RÉSUMÉS}

Souvent exclus des projets de développement urbain et de renforcement économique, les femmes habitant les quartiers squattés de la ville d'El-Mina, Tripoli, Liban Nord, arrivent à accéder aux soins requis malgré les vulnérabilités dont elles souffrent. Des études précédentes ont montré que l'accessibilité aux soins des populations pauvres est limitée par le manque des ressources économiques. La présente étude explore le comportement des femmes dans la recherche du soin au cours de la phase périnatale ainsi que pour leurs enfants âgés de moins de cinq ans. Les résultats obtenus mettent en évidence un contexte de solidarité régnant dans la ville qui contribue à l'atténuation des effets des facteurs géographiques, sociaux, culturels et économiques, caractérisant le milieu habité, sur la santé périnatale tout en favorisant l'accessibilité aux soins nécessaires. La présence de plusieurs acteurs urbains assurant des offres multiples et une prise en charge des soins primaires marque ce réseau de solidarité. Cependant, la qualité du soin, privilégiée au cours de la grossesse, est remplacée par le recours à l'automédication pour les enfants qui souffrent des problèmes de santé.

Often excluded from urban development and economic empowerment projects, the squatter settlements of El-Mina city, Tripoli, Northern Lebanon, suffer from multiple vulnerabilities. The latter embrace precarious housing, flooding of streets and housing, unmanaged wastewater and solid waste, and foul odors, as declared by the permanent residents. This classifies these territories, Hay El-Tanak, Haouch, and El Masaken El-Chaabiyah as the most underprivileged and destitute of El-Mina city and reflects a disadvantaged population profile. Previous studies have shown that the accessibility to healthcare centers of the deprived populations is limited due to the deficiency in economic resources and the lack of adequate infrastructures. However, in the above mentioned territories, the women inhabitants can access health care needed despite the vulnerabilities they suffer from.

The current study is realized on a local large scale of these settlements favoring the exploration of women's health profile during « perinatal » phase and children's health status until the age of five.

A field survey was conducted in the three squatter settlements targeting 139 women in the age of reproduction who revealed information about their health and their families' health. Aiming to understand the behavior of women seeking healthcare, we calculated the accessibility Index inspired by Shimbel method and then compared the values obtained with the actual conduct declared during the survey carried out in the three squatter settlements targeted. All the health centers studied were geographically located in order to see their positioning relatively to the three settlements studied using the geographic travel distance from each settlement to every center mentioned.

The analysis showed that the geographic proximity has no main impact on the destinations adopted by the pregnant women who were included in the samples studied. Even though residing in deprived urban territories, a high percentage of women (77 \% at the El-Masaken El-Chaabiyah, $61 \%$ at Haouch, and $56 \%$ at Hay El-Tanak) have a tendency to constantly monitor their pregnancy, visiting the gynecologist on a regular basis starting from the first month. Several factors guide these choices which are related to the quality of healthcare, the trust towards the specialist and also to the cost of the services received. 
For their children's health care, and when diarrhea, fever, and coughing symptoms emerge, mothers take on different preferences. These are also adopted to seek children's vaccination.

Considered as key role, the solidarity context reigning in the city contributes in alleviating the effects of the living territory on the health of informal settlements' women's health during the perinatal phase. This network of solidarity engages many urban actors of the health sector who aim to facilitate the accessibility of poor populations to health care and aspire to address the lack of universal health coverage not provided by the State. These include dispensaries, hospitals, doctors, non-governmental organizations, charity religious and political associations. In 2012 an assistance model was operating in El-Mina city. This model partially replaced the social security coverage and the private health insurance which exclude the disadvantaged unemployed population occupying squatter settlements. The multiple healthcare offers available in El-Mina city and the presence of several urban actors enabled this accessibility. However, the quality of healthcare sought during the prenatal phase is substituted by auto-medication when children aged under five get ill. It is important to note that as long as the vulnerabilities persist these actions remain incomplete.

\section{INDEX}

Mots-clés : quartiers squattés, accessibilité, vulnérabilités, acteurs urbains, santé périnatale Index géographique : Liban, El Mina, Tripoli

Keywords : squatter settlements, accessibility, vulnerabilities, urban actors, perinatal health

\section{AUTEUR}

\section{ELIAS ALISSAR}

Docteur (Géographie de la Santé), Université Paris-Est Créteil et Université Saint Joseph, Beyrouth 